\title{
Some periodic and fixed point theorems on quasi- $b$-gauge spaces
}

\author{
N. Zikria' ${ }^{1}$ M. Samreen ${ }^{1}$, T. Kamran ${ }^{1}$, H. Aydi ${ }^{2,3,4^{*}}$ (D) and C. Park ${ }^{5^{*}}$
}

\author{
"Correspondence: \\ hassen.aydi@isima.rnu.tn; \\ baak@hanyang.ac.kr \\ ${ }^{2}$ Institut Supérieur d'Informatique et \\ des Techniques de Communication, \\ Université de Sousse, $\mathrm{H}$. Sousse, \\ 4000, Tunisia \\ ${ }^{5}$ Research Institute for Natural \\ Sciences, Hanyang University, Seoul \\ 04763, Korea \\ Full list of author information is \\ available at the end of the article
}

\begin{abstract}
The notions of a quasi-b-gauge space $\left(U, Q_{s ; \Omega}\right)$ and a left (right) $\mathcal{J}_{s ; \Omega}$-family of generalized quasi-pseudo-b-distances generated by $\left(U, Q_{s ;} \Omega\right)$ are introduced. Moreover, by using this left (right) $\mathcal{J}_{s ; \Omega}$-family, we define the left (right) $\mathcal{J}_{s: \Omega}$-sequential completeness, and we initiate the Nadler type contractions for set-valued mappings $T: U \rightarrow C / \mathcal{J}_{s ; \Omega}(U)$ and the Banach type contractions for single-valued mappings $T: U \rightarrow U$, which are not necessarily continuous. Furthermore, we develop novel periodic and fixed point results for these mappings in the new setting, which generalize and improve the existing fixed point results in the literature. Examples validating our obtained results are also given.
\end{abstract}

MSC: $47 \mathrm{H} 10 ; 54 \mathrm{H} 25$

Keywords: Generalized quasi-pseudo-b-distance; Banach contraction; Nadler contraction; Quasi-b-gauge space; Fixed point; Periodic point

\section{Introduction}

For $U \neq \emptyset$, let $2^{U}$ indicate the set of all nonempty subsets of the space $U$. If $T: U \rightarrow 2^{U}$ is a set-valued mapping, then $\operatorname{Fix}(T)=\{u \in U: u \in T(u)\}$ denotes the set of all fixed points of $T$, and $\operatorname{Per}(T)=\left\{u \in U: u \in T^{[k]}(u)\right.$ for some $k$ in $\left.\mathbb{N}\right\}$ denotes the set of all periodic points of $T$, where $T^{[k]}=T \circ T \circ T \circ \cdots \circ T$ (k-times). A dynamic process of the system $(U, T)$ starting at $z^{0} \in U$, is a sequence $\left\{z^{m}: m \in\{0\} \cup \mathbb{N}\right\}$ described by $z^{m} \in T\left(z^{m-1}\right)$ for all $m \in \mathbb{N}$.

Recall that for a map $T: U \rightarrow U$, a sequence $\left(z^{m}: m \in\{0\} \cup \mathbb{N}\right)$ beginning at $z^{0} \in U$ such that $z^{m}=T^{[m]}\left(z^{0}\right)$ for all $m \in\{0\} \cup \mathbb{N}$ is called a Picard iteration.

In 1966, Dugundji [1] initiated the idea of gauge spaces, which generalizes metric spaces (or more generally, pseudo-metric spaces). Gauge spaces have the characteristic that even the distance between two distinct points of the space may be zero. This simple characterization has been the center of interest for many researchers worldwide. In 1973, Reilly [2] initiated quasi-gauge spaces. According to his definition, quasi-gauge spaces generalize quasi-pseudo metric space by replacing a single quasi-pseudo metric space with the family of such spaces on the set. In this way, he was also able to show that quasi-gauge spaces generalize gauge spaces. In 2015, Ali et al. [3] introduced the concept of $b$-gauge spaces and obtained some fixed point results. For further facts on gauge spaces, we recommend

(c) The Author(s) 2022. This article is licensed under a Creative Commons Attribution 4.0 International License, which permits use, sharing, adaptation, distribution and reproduction in any medium or format, as long as you give appropriate credit to the original author(s) and the source, provide a link to the Creative Commons licence, and indicate if changes were made. The images or other third party material in this article are included in the article's Creative Commons licence, unless indicated otherwise in a credit line to the material. If material is not included in the article's Creative Commons licence and your intended use is not permitted by statutory regulation or exceeds the permitted use, you will need to obtain permission directly from the copyright holder. To view a copy of this licence, visit http://creativecommons.org/licenses/by/4.0/. 
the readers to Agarwal et. al. [4], Frigon [5], Chis and Precup [6], Chifu and Petrusel [7], Lazara and Petrusel [8], Cherichi et al. [9, 10] and Jleli et al. [11].

For a long time, it was unknown how to define the distances, which generalize metrics, or pseudo metrics. These distances provide important and powerful tools in finding the solutions to various crucial problems in fixed point theory. In this direction, the works done by Kada et al. [12], Suzuki [13], and Lin and Du [14] in metric spaces are appreciable.

Wlodarczyk and Plebaniak [15] introduced the notion of left (right) $\mathcal{J}$-families of generalized quasi-pseudo distances in quasi-gauge spaces, which generalized the abovementioned distances and provided useful and important aid for solving numerous problems of nonlinear analysis. To know how these families work and help in proving different results of fixed point theory, see [16-21].

The famous fixed point results due to Banach [22] called the Banach contraction principle has attained its fame in the case of single-valued mappings and attracted various authors for many years. The principle assures the uniqueness and existence of a fixed point of certain self-maps on a complete metric space and gives a powerful tool to estimate the fixed point. Nadler [23] extended the Banach contraction principle to the case of multivalued mappings using the idea of a Hausdorff metric. Their analogs in more general spaces are important, fascinating and challenging for most researchers. For further results on the subject, see [24-26].

This paper aims to introduce the notions of a quasi- $b$-gauge space $\left(U, Q_{s ; \Omega}\right)$ and left (right) $\mathcal{J}_{s ; \Omega}$-families of generalized quasi-pseudo- $b$-distances generated by $\left(U, Q_{s ; \Omega}\right)$. Moreover, by using these left (right) $\mathcal{J}_{s ; \Omega}$-families, we introduce the concept of left (right) $\mathcal{J}_{s ; \Omega}$-sequential completeness. We also investigate the Nadler type contractions for setvalued maps $T: U \rightarrow \mathrm{Cl}^{\mathcal{J}_{s ; \Omega}}(U)$ and Banach type contractions for single-valued maps $T: U \rightarrow U$ (that are not necessarily continuous). Furthermore, we present new periodic and fixed point results for such mappings in the new setting, which generalize and complement the existing fixed point results in he literature. Some examples are also provided in support of the main results.

\section{Preliminaries}

The following concepts are useful in the entire paper. The famous Banach contraction principle [22] states that:

Let $(U, q)$ be a complete metric space. Suppose that $T: U \rightarrow U$ is a contraction mapping, i.e., there exists $\mu \in[0,1)$ such that

$$
q(T e, T f) \leq \mu q(e, f)
$$

for all $e, f \in U$. Then

(i) $T$ has a unique fixed point $g$ in $U$;

(ii) for each $g^{0} \in U$, the sequence $\left\{g^{m}=T^{[m]}\left(g^{0}\right): m \in \mathbb{N}\right\}$ converges to such a fixed point.

Recall that the Hausdorff metric $H^{q}$ on the class of all nonempty closed and bounded subsets $C B(U)$ in the metric space $(U, q)$ is described for all $A, B \in C B(U)$ in the following 
way

$$
H^{q}(A, B)=\max \left\{\sup _{a \in A} q(a, B), \sup _{b \in B} q(b, A)\right\}
$$

where $q(a, B)=\inf _{b \in B} q(a, b)$, for $a \in U$.

The main result of Nadler [23] for set-valued mappings is:

Let $(U, q)$ be a complete metric space and let $T: U \rightarrow C B(U)$ satisfy a $\left(H^{q}, \mu\right)$ contraction, i.e., there exists $\mu \in[0,1)$ such that

$$
H^{q}(T(e), T(f)) \leq \mu q(e, f)
$$

for all $e, f \in U$. Then there is $g \in U$ such that $g \in T(g)$ (that is, $\operatorname{Fix}(T) \neq \emptyset)$.

On the other hand, Bakhtin [27] introduced the notion of a $b$-metric space in 1989, and Czerwik [28] presented it formally in 1993 in order to generalize the Banach contraction principle.

Definition 2.1 ([27]) Let $U$ be a nonempty set and $s \geq 1$. A function $q: U \times U \rightarrow[0, \infty)$ is a $b$-metric if it satisfies the following for all $e, f, g \in U$ :

(a) $q(e, f)=0 \Leftrightarrow e=f$;

(b) $q(e, f)=q(f, e)$;

(c) $q(e, g) \leq s\{q(e, f)+q(f, g)\}$.

The pair $(U, q)$ is said to be a $b$-metric space.

Example 2.2 Suppose $U=[0,1]$. Define $q: U \times U \rightarrow[0, \infty)$ for all $e, f \in U$ as:

$$
q(e, f)=(e-f)^{2} \text {. }
$$

Then $q$ is a $b$-metric on $U$, where $s=2$.

We observe that $q$ is not a metric on $U$, since the triangular inequality does not hold.

Also, we note from the definition of a $b$-metric space that when $s=1$, both the concepts of a metric space and a $b$-metric space coincide. Thus, the class of $b$-metric spaces is bigger than the class of metric spaces.

The references [29-39] can be seen for other definitions and results in the setting of $b$-metric spaces.

Definition 2.3 ([40]) Suppose $U$ is a non-void set. The function $q: U \times U \rightarrow[0, \infty)$ is called a quasi-pseudo metric if it fulfils the following for all $e, f, g \in U$ :

(a) $q(e, e)=0$

(b) $q(e, g) \leq q(e, f)+q(f, g)$.

The pair $(U, q)$ is called a quasi-pseudo metric space.

Example 2.4 Let $U=\mathbb{R}$ and define $q: U \times U \rightarrow[0, \infty)$ by

$$
q(e, f)= \begin{cases}|e-f| & \text { if } e<f, \\ 0 & \text { if } e \geq f .\end{cases}
$$

Then $q$ is a quasi-pseudo metric on $U$ and $(U, q)$ is a quasi-pseudo metric space. 
Since the symmetric property does not hold (i.e., $q(e, f) \neq d(f, e)),(U, q)$ is not a pseudo metric space, and hence it is not a metric space.

\section{Main results}

In 2015, Ali et al. [3] has defined gauge spaces in the local of $b_{s}$-pseudo metrics, called $b$-gauge spaces. In order to introduce quasi- $b$-gauge spaces, we start the introduction of the notion of a quasi-pseudo- $b$ metric.

Definition 3.1 Let $U$ be a nonempty set and $s \geq 1$. The map $q: U \times U \rightarrow[0, \infty)$ is called to be a quasi-pseudo- $b$ metric if it satisfies the following for all $e, f, g \in U$ :

(a) $q(e, e)=0$;

(b) $q(e, g) \leq s\{q(e, f)+q(f, g)\}$.

The pair $(U, q)$ is called a quasi-pseudo- $b$ metric space. A Hausdorff quasi-pseudo- $b$ metric space $(U, q)$ satisfies

$$
e \neq f \quad \Rightarrow \quad q(e, f)>0 \vee q(f, e)>0
$$

for all $e, f \in U$.

Example 3.2 Let $U=l_{p}=\left\{\left\{x_{n}\right\}_{n \geq 1} \subset \mathbb{R}, \sum_{n=1}^{\infty}\left|x_{n}\right|^{p}<\infty\right\}$, where $1 \leq p<\infty$. Define $q: U \times$ $U \rightarrow[0, \infty)$ for all $x, y \in U$ by

$$
q(x, y)= \begin{cases}0 & \text { if } x \leq y, \\ \left(\sum_{n=1}^{\infty}\left|x_{n}\right|^{p}\right)^{\frac{1}{p}} & \text { if } x>y .\end{cases}
$$

Then $q$ is a quasi-pseudo- $b$-metric on $U$ with $s=p \geq 1$. Since symmetry property does not hold, $q$ is not a pseudo- $b$-metric, and hence it is not a $b$-metric.

Example 3.3 Suppose $U=[0,6]$. Define $q: U \times U \rightarrow[0, \infty)$ for all $e, f \in U$ by

$$
q(e, f)= \begin{cases}0 & \text { if } e \geq f, \\ (e-f)^{2} & \text { if } e<f .\end{cases}
$$

Then $q$ is a quasi-pseudo- $b$-metric on $U$. Indeed, $q(e, e)=0$ for all $e \in U$. Further, $q(e, g) \leq$ $2\{q(e, f)+q(f, g)\}$ holds for all $e, f, g \in U$ and for $s=2$. Also, $(U, q)$ is a Hausdorff quasipseudo-b-metric space.

Definition 3.4 Each family $Q_{s ; \Omega}=\left\{q_{\beta}: \beta \in \Omega\right\}$ of quasi-pseudo- $b$ metrics $q_{\beta}: U \times U \rightarrow$ $[0, \infty)$ for $\beta \in \Omega$ is said to be a quasi- $b$-gauge on $U$.

Definition 3.5 The family $Q_{s ; \Omega}=\left\{q_{\beta}: \beta \in \Omega\right\}$ is called to be separating if for every pair $(e, f)$, where $e \neq f$, there exists $q_{\beta} \in Q_{s ; \Omega}$ such that either $q_{\beta}(e, f)>0$ or $q_{\beta}(f, e)>0$.

Definition 3.6 Let the family $Q_{s ; \Omega}=\left\{q_{\beta}: \beta \in \Omega\right\}$ be a quasi- $b$-gauge on $U$. The topology $\mathcal{T}\left(Q_{s ; \Omega}\right)$ whose subbase is defined by the family $\mathcal{B}\left(Q_{s ; \Omega}\right)=\left\{B\left(e, \epsilon_{\beta}\right): e \in U, \epsilon_{\beta}>0, \beta \in \Omega\right\}$ of all balls $B\left(e, \varepsilon_{\beta}\right)=\left\{f \in U: q_{\beta}(e, f)<\epsilon_{\beta}\right\}$ and is called the topology induced by $Q_{s ; \Omega}$ on $U$. 
Definition 3.7 Suppose $(U, \mathcal{T})$ is a topological space and $Q_{s ; \Omega}$ is a quasi-b-gauge on $U$ such that $\mathcal{T}=\mathcal{T}\left(Q_{s ; \Omega}\right)$. Then the topological space $\left(U, Q_{s ; \Omega}\right)$ is called to be a quasi- $b$-gauge space. We note that $\left(U, Q_{s ; \Omega}\right)$ is the Hausdorff if $Q_{s ; \Omega}$ is separating.

\section{Remark 3.8}

(a) Each topological space and quasi-uniform space is a quasi-gauge space [2]. Also, each quasi-gauge space is a quasi- $b$-gauge space (for $s=1$ ). Therefore, in the asymmetric structure, we can term a quasi- $b$-gauge space as the largest general space.

(b) We observe that if $s=1$, the above definitions turn down to agree with the definitions in quasi-gauge spaces.

We now establish the notion of left (right) $\mathcal{J}_{s ; \Omega}$-families of generalized quasi-pseudo- $b$ distances on $U$ [left (right) $\mathcal{J}_{s ; \Omega}$-families are the generalizations of quasi- $b$-gauges].

Definition 3.9 Let $\left(U, Q_{s ; \Omega}\right)$ be a quasi-b-gauge space. The family $\mathcal{J}_{s ; \Omega}=\left\{J_{\beta}: \beta \in \Omega\right\}$ where $J_{\beta}: U \times U \rightarrow[0, \infty), \beta \in \Omega$ is called the left (right) $\mathcal{J}_{s ; \Omega}$-family of generalized quasipseudo- $b$-distances on $U$ (for short, left (right) $\mathcal{J}_{s ; \Omega}$-family on $U$ ) if the following statements hold for all $\beta \in \Omega$ and for all $x, y, z \in U$ :

$(\mathcal{J} 1) J_{\beta}(x, z) \leq s_{\beta}\left\{J_{\beta}(x, y)+J_{\beta}(y, z)\right\}$;

$(\mathcal{J} 2)$ for sequences $\left(u_{m}: m \in \mathbb{N}\right)$ and $\left(v_{m}: m \in N\right)$ in $U$ fulfilling

$$
\begin{aligned}
& \lim _{m \rightarrow \infty} \sup _{n>m} J_{\beta}\left(u_{m}, u_{n}\right)=0, \\
& \left(\lim _{m \rightarrow \infty} \sup _{n>m} J_{\beta}\left(u_{n}, u_{m}\right)=0\right),
\end{aligned}
$$

and

$$
\begin{aligned}
& \lim _{m \rightarrow \infty} J_{\beta}\left(v_{m}, u_{m}\right)=0, \\
& \left(\lim _{m \rightarrow \infty} J_{\beta}\left(u_{m}, v_{m}\right)=0\right),
\end{aligned}
$$

the following hold:

$$
\lim _{m \rightarrow \infty} q_{\beta}\left(v_{m}, u_{m}\right)=0
$$

and

$$
\left.\left(\lim _{m \rightarrow \infty} q_{\beta}\left(u_{m}, v_{m}\right)=0\right)\right)
$$

We denote

$$
\mathbb{J}_{\left(U, Q_{s ; \Omega}\right)}^{L}=\left\{\mathcal{J}_{s ; \Omega}: \mathcal{J}_{s ; \Omega}=\left\{J_{\beta}: \beta \in \Omega\right\} \text { is a left } \mathcal{J}_{s ; \Omega} \text {-family on } U\right\}
$$

and

$$
\left.\mathbb{J}_{\left(U, Q_{s ; \Omega}\right)}^{R}=\left\{\mathcal{J}_{s ; \Omega}: \mathcal{J}_{s ; \Omega}=\left\{J_{\beta}: \beta \in \Omega\right\}\right\} \text { is a right } \mathcal{J}_{s ; \Omega} \text {-family on } U\right\} \text {. }
$$


Now, we mention some trivial properties of left (right) $\mathcal{J}_{s ; \Omega}$-families.

Remark 3.10 Let $\left(U, Q_{s ; \Omega}\right)$ be a quasi- $b$-gauge space.

(a) $Q_{s ; \Omega} \in \mathbb{J}_{\left(U, Q_{s ; \Omega}\right)}^{L} \cap \mathbb{J}_{\left(U, Q_{s ; \Omega}\right)}^{R}$.

(b) Let $\mathcal{J}_{s ; \Omega} \in \mathbb{J}_{\left(U, Q_{s ; \Omega}\right)}^{L}$ or $\mathcal{J}_{s ; \Omega} \in \mathbb{J}_{\left(U, Q_{s ; \Omega}\right)}^{R}$. If $J_{\beta}(v, v)=0$ for all $\beta \in \Omega$ and for all $v \in U$, then for each $\beta \in \Omega, J_{\beta}$ is a quasi-pseudo- $b$ metric.

(c) There is an example of $\mathcal{J}_{s ; \Omega} \in \mathbb{J}_{\left(U, Q_{s ; \Omega}\right)}^{L}$ and $\mathcal{J}_{s ; \Omega} \in \mathbb{J}_{\left(U, Q_{s ; \Omega}\right)}^{R}$, which shows that the maps $J_{\beta}, \beta \in \Omega$ are not quasi-pseudo- $b$ metric (see Example 3.12 below).

(d) We note that if $s=1$, the above definition reduces to the corresponding definition in quasi-gauge spaces.

Proposition 3.11 Let $\left(U, Q_{s ; \Omega}\right)$ be the Hausdorff quasi-b-gauge space. Take the family $\mathcal{J}_{s ; \Omega}=\left\{J_{\beta}: \beta \in \Omega\right\}$ to be the left (right) $\mathcal{J}_{s ; \Omega}$-family of generalized quasi-pseudo-b-distances on $U$. Then there is $\beta \in \Omega$ such that

$$
e \neq f \quad \Rightarrow \quad J_{\beta}(e, f)>0 \vee J_{\beta}(f, e)>0
$$

for all $e, f \in U$.

Proof By incorporating the definition of the left (right) $\mathcal{J}_{s ; \Omega}$-family of generalized quasipseudo- $b$-distances on $U$ in the proof of Proposition 3.11 of [15], the proof of our result can easily be obtained.

Example 3.12 Let $\left(U, Q_{s ; \Omega}\right)$ be a quasi-b-gauge space, where $U$ contains at least two distinct points and $Q_{s ; \Omega}=\left\{q_{\beta}: \beta \in \Omega\right\}$ is the family of quasi-pseudo- $b$ metrics $q_{\beta}: U \times U \rightarrow$ $[0, \infty), \beta \in \Omega$.

Let the set $F \subset U$ contain at least two distinct, arbitrary and fixed points, and let $d_{\beta} \in$ $(0, \infty), \beta \in \Omega$ satisfy $\delta_{\beta}(F)<d_{\beta}$ for all $\beta \in \Omega$, where $\delta_{\beta}(F)=\sup \left\{q_{\beta}(u, v): u, v \in F\right\}$ for all $\beta \in \Omega$. Let the family $\mathcal{J}_{s ; \Omega}=\left\{J_{\beta}: \beta \in \Omega\right\}$ of maps $J_{\beta}: U \times U \rightarrow[0, \infty), \beta \in \Omega$ be defined for all $e, f \in U$ as:

$$
J_{\beta}(e, f)= \begin{cases}q_{\beta}(e, f) & \text { if } F \cap\{e, f\}=\{e, f\} \\ d_{\beta} & \text { if } F \cap\{e, f\} \neq\{e, f\}\end{cases}
$$

Then $\mathcal{J}_{\varphi ; \Omega} \in \mathbb{J}_{(U, Q)}^{L} \cap \mathbb{J}_{(U, Q)}^{R}$.

We notice that $J_{\beta}(e, g) \leq \frac{d_{\beta}}{\delta_{\beta}(F)}\left\{J_{\beta}(e, f)+J_{\beta}(f, g)\right\}$ for all $\beta \in \Omega$ and for all $e, f, g \in U$, where $\frac{d_{\beta}}{\delta_{\beta}(F)}=s_{\beta}>1, \beta \in \Omega$. Thus, the condition $\left(\mathcal{J}_{1}\right)$ holds. Indeed, the condition $\left(\mathcal{J}_{1}\right)$ does not hold only if there are some $\beta \in \Omega$ and $e, f, g \in U$ such that $J_{\beta}(e, g)=d_{\beta}, J_{\beta}(e, f)=q_{\beta}(e, f)$, $J_{\beta}(f, g)=q_{\beta}(f, g)$ and $s_{\beta}\left\{q_{\beta}(e, f)+q_{\beta}(f, g)\right\} \leq d_{\beta}$. However, this implies that there exists $h \in\{e, g\}$ such that $h \notin F$ and, on the other hand, $e, f, g \in F$, which is impossible.

Now, suppose that the sequences $\left\{u_{m}\right\}$ and $\left\{v_{m}\right\}$ in $U$ are satisfying (3.4) and (3.6). Then in particular (3.6) yields that there exists $m_{1}=m_{1}(\beta) \in \mathbb{N}$ such that for all $m \geq m_{1}$, for all $\beta \in \Omega$, and for all $0<\epsilon<d_{\beta}$, we have

$$
J_{\beta}\left(v_{m}, u_{m}\right)<\epsilon
$$


By (3.11) and (3.10), denoting $m^{\prime}=\min \left\{m_{1}(\beta): \beta \in \Omega\right\}$, we have for all $m \geq m^{\prime}$

$$
F \cap\left\{v_{m}, u_{m}\right\}=\left\{v_{m}, u_{m}\right\} .
$$

Let there exist $m^{\prime} \in \mathbb{N}$ such that for all $m \geq m^{\prime}$, for all $\beta \in \Omega$, and for all $0<\epsilon<d_{\beta}$, we have

$$
q_{\beta}\left(v_{m}, u_{m}\right)=J_{\beta}\left(v_{m}, u_{m}\right)<\epsilon
$$

Hence, the sequences $\left\{u_{m}\right\}$ and $\left\{v_{m}\right\}$ satisfy (3.8). Therefore, $\mathcal{J}_{s ; \Omega}$ is a left $\mathcal{J}_{s ; \Omega}$-family.

Similarly, we show that if $\left\{u_{m}\right\}$ and $\left\{v_{m}\right\}$ in $U$ satisfy (3.5) and (3.7), then (3.9) holds, and thus $\mathcal{J}_{s ; \Omega}$ is a right $\mathcal{J}_{s ; \Omega}$-family.

Now, using the left (right) $\mathcal{J}_{s ; \Omega}$-family on $U$, we define the left (right) $\mathcal{J}_{s ; \Omega}$-completeness in the quasi-b-gauge space $\left(U, Q_{s ; \Omega}\right)$.

Definition 3.13 Let $\left(U, Q_{s ; \Omega}\right)$ be a quasi- $b$-gauge space, and let $\mathcal{J}_{s ; \Omega}=\left\{J_{\beta}: \beta \in \Omega\right\}$ be a left (right) $\mathcal{J}_{s ; \Omega}$-family on $U$.

(A) A sequence $\left\{u_{m}\right\}_{m \in \mathbb{N}}$ is said to be a left (right) $\mathcal{J}_{s ; \Omega}$-Cauchy sequence in $U$ if for all $\beta \in \Omega$, we have

$$
\lim _{m \rightarrow \infty} \sup _{n>m} J_{\beta}\left(u_{m}, u_{n}\right)=0 \quad\left(\lim _{m \rightarrow \infty} \sup _{n>m} J_{\beta}\left(u_{n}, u_{m}\right)=0\right) .
$$

(B) A sequence $\left\{u_{m}\right\}_{m \in \mathbb{N}}$ is said to be the left (right) $\mathcal{J}_{s ; \Omega}$-convergent to $u \in U$ if $\lim _{m \rightarrow \infty}^{L-\mathcal{J}_{s} \Omega} u_{m}=u\left(\lim _{m \rightarrow \infty}^{R-\mathcal{J}_{s ; \Omega}} u_{m}=u\right)$, where $\lim _{m \rightarrow \infty}^{L-\mathcal{J}_{s ; \Omega}} u_{m}=u \Leftrightarrow \forall \forall_{\beta \in \Omega}\left\{\lim _{m \rightarrow \infty} J_{\beta}\left(u, u_{m}\right)=0\right\}$ $\left(\lim _{m \rightarrow \infty}^{R-\mathcal{J}_{s ; \Omega}} u_{m}=u \Leftrightarrow \forall \beta \in \Omega\left\{\lim _{m \rightarrow \infty} J_{\beta}\left(u_{m}, u\right)=0\right\}\right)$.

(C) If $S_{\left(u_{m}: m \in \mathbb{N}\right)}^{L-\mathcal{J}_{s ; \Omega}} \neq \emptyset\left(S_{\left(u_{m}: m \in \mathbb{N}\right)}^{R-\mathcal{J}_{s ; \Omega}} \neq \emptyset\right)$, where $S_{\left(u_{m}: m \in \mathbb{N}\right)}^{L-\mathcal{J}_{s ; \Omega}}=\left\{u \in U: \lim _{m \rightarrow \infty}^{L-\mathcal{J}_{s ; \Omega}} u_{m}=u\right\}$ $\left(S_{\left(u_{m}: m \in \mathbb{N}\right)}^{R-\mathcal{J}_{s ; \Omega}}=\left\{u \in U: \lim _{m \rightarrow \infty}^{R-\mathcal{J}_{s ; \Omega}} u_{m}=u\right\}\right)$. Then the sequence $\left\{u_{m}\right\}_{m \in \mathbb{N}}$ in $U$ is the left (right) $\mathcal{J}_{s ; \Omega}$-convergent in $U$.

(D) The space $\left(U, Q_{s ; \Omega}\right)$ is a left (right) $\mathcal{J}_{s ; \Omega}$-sequentially complete quasi- $b$-gauge space if each left (right) $\mathcal{J}_{s ; \Omega}$-Cauchy sequence in $U$ is left (right) $\mathcal{J}_{s ; \Omega}$-convergent in $U$.

Definition 3.14 Suppose $\left(U, Q_{s ; \Omega}\right)$ is a quasi-b-gauge space, and let $T: U \rightarrow 2^{U}$ be a set valued map. The map $T^{[k]}$ (for $k \in \mathbb{N}$ ) is called a left (right) $Q_{s ; \Omega}$-quasi-closed map on $U$ if for each sequence $\left\{z_{m}\right\}_{m \in \mathbb{N}}$ within $T^{[k]}(U)$, which is left (right) $Q_{s ; \Omega}$-convergent in $U$, thus $S_{\left(z_{m}: m \in \mathbb{N}\right)}^{L-Q_{s ; \Omega}} \neq \emptyset\left(S_{\left(z_{m}: m \in \mathbb{N}\right)}^{R-Q_{s}} \neq \emptyset\right)$, having $\left\{x_{m}\right\}_{m \in \mathbb{N}}$ and $\left\{y_{m}\right\}_{m \in \mathbb{N}}$ as its subsequences satisfying $y_{m} \in T^{[k]}\left(x_{m}\right)$ for all $m \in \mathbb{N}$, has the property that there exists $z \in S_{\left(z_{m}: m \in \mathbb{N}\right)}^{L-Q_{s ; \Omega}}\left(z \in S_{\left(z_{m}: m \in \mathbb{N}\right)}^{R-Q_{s ; \Omega}}\right)$ such that $z=T^{[k]}(z)\left(z=T^{[k]}(z)\right)$.

Remark 3.15 Suppose $\left(U, Q_{s ; \Omega}\right)$ is a quasi-b-gauge space.

(a) If $\left\{u_{m}: m \in \mathbb{N}\right\}$ is a left (right) $\mathcal{J}_{s ; \Omega}$-convergent sequence in $U$, then for every subsequence $\left\{v_{m}\right\}_{m \in \mathbb{N}}$, we have

$$
S_{\left(u_{m}: m \in \mathbb{N}\right)}^{L-\mathcal{J}_{s ; \Omega}} \subset S_{\left(v_{m}: m \in \mathbb{N}\right)}^{L-\mathcal{J}_{s ; \Omega}} \quad\left(S_{\left(u_{m}: m \in \mathbb{N}\right)}^{R-\mathcal{J}_{s ; \Omega}} \subset S_{\left(v_{m}: m \in \mathbb{N}\right)}^{R-\mathcal{J}_{s ; \Omega}}\right)
$$

(b) We observe that if $s=1$ for all $\beta \in \Omega$, the above definitions turn down to agree with the definitions in quasi-gauge spaces. 
Definition 3.16 Let $\left(U, Q_{s ; \Omega}\right)$ be a quasi- $b$-gauge space. Let $\mathcal{J}_{s ; \Omega}=\left\{J_{\beta}: \beta \in \Omega\right\}$ be a left (right) $\mathcal{J}_{s ; \Omega}$-family on $U$. The map $T: U \rightarrow 2^{U}$ is the left (right) $\mathcal{J}_{s ; \Omega}$-admissible at a point $z^{0} \in U$ if for any sequence $\left\{z^{m}: m \in\{0\} \cup \mathbb{N}\right\}$ satisfying $z^{m+1} \in T\left(z^{m}\right)$ for all $m \in\{0\} \cup \mathbb{N}$ and $\lim _{m \rightarrow \infty} \sup _{n>m} J_{\beta}\left(z^{m}, z^{n}\right)=0\left(\lim _{m \rightarrow \infty} \sup _{n>m} J_{\beta}\left(z^{n}, z^{m}\right)=0\right)$ for all $\beta \in \Omega$, there exists $z \in U$ such that for all $\beta \in \Omega, \lim _{m \rightarrow \infty} J_{\beta}\left(z, z^{m}\right)=0\left(\lim _{m \rightarrow \infty} J_{\beta}\left(z^{m}, z\right)=0\right)$.

The set-valued map $T: U \rightarrow 2^{U}$ is the left (right) $\mathcal{J}_{s ; \Omega}$-admissible in $U$ if $T: U \rightarrow 2^{U}$ is the left (right) $\mathcal{J}_{s ; \Omega}$-admissible at each point $z^{0} \in U$.

Remark 3.17 Suppose $\left(U, Q_{s ; \Omega}\right)$ is a quasi- $b$-gauge space, and let $\mathcal{J}_{s ; \Omega}=\left\{J_{\beta}: \beta \in \Omega\right\}$ be a left (right) $\mathcal{J}_{s ; \Omega}$-family on $U$.

(a) If $(U, Q)$ is left (right) $\mathcal{J}_{s ; \Omega}$-sequentially complete, then $T: U \rightarrow 2^{U}$ is the left (right) $\mathcal{J}_{s ; \Omega}$-admissible on $U$.

(b) If $s=1$, the above definitions reduce to the corresponding definition in quasi-gauge spaces.

Definition 3.18 Let $\left(U, Q_{s ; \Omega}\right)$ be a quasi $b$-gauge space, and let $\mathcal{J}_{s ; \Omega}=\left\{J_{\beta}: \beta \in \Omega\right\}$ be the a left (right) $\mathcal{J}_{s ; \Omega}$-family on $U$. A set $W \in 2^{U}$ is the left (right) $\mathcal{J}_{s ; \Omega}$-closed in $U$ if $W=c l_{U}^{L-\mathcal{J}_{s ; \Omega}}(W)\left(W=c l_{U}^{R-\mathcal{J}_{s ; \Omega}}(W)\right)$, where $c l_{U}^{L-\mathcal{J}_{s ; \Omega}}(W)\left(c l_{U}^{R-\mathcal{J}_{s ; \Omega}}(W)\right)$, is the left (right) $\mathcal{J}_{s ; \Omega}$-closure in $U$ and is defined by $c l_{U}^{L-\mathcal{J}_{s ; \Omega}}(W)=\left\{z \in U: \lim _{m \rightarrow \infty}^{L-\mathcal{J}_{s ; \Omega}} z_{m}=z\right\}$

$\left(c l_{U}^{R-\mathcal{J}_{s ; \Omega}}(W)=\left\{z \in U: \lim _{m \rightarrow \infty}^{R-\mathcal{J}_{s ; \Omega}} z_{m}=z\right\}\right)$.

Define $C l^{L-\mathcal{J}_{s ; \Omega}(U)}=\left\{W \in 2^{U}: W=c l_{U}^{L-\mathcal{J}_{s ; \Omega}}(W)\right\}\left(C l^{R-\mathcal{J}_{s ; \Omega}}(U)=\left\{W \in 2^{U}: W=\right.\right.$ $\left.\left.c l_{U}^{R-\mathcal{J}_{s ; \Omega}}(W)\right\}\right)$. Thus, $C l^{L-\mathcal{J}_{s ; \Omega}}(U)\left(C l^{R-\mathcal{J}_{s ; \Omega}}(U)\right)$ symbolizes the class of all non-empty left (right) $\mathcal{J}_{s ; \Omega}$-closed subsets of $U$.

Remark 3.19 We note that if $s=1$, the above definition reduces to the corresponding definition in quasi-gauge spaces.

In a quasi- $b$-gauge space, we describe the left (right) Hausdorff type quasi- $b$-distances and Nadler type left (right) contractions in the following way.

Definition 3.20 Let $\left(U, Q_{s ; \Omega}\right)$ be a quasi- $b$-gauge space, and let $\mathcal{J}_{s ; \Omega}=\left\{J_{\beta}: \beta \in \Omega\right\}$ be a left (right) $\mathcal{J}_{s ; \Omega}$-family on $U$. Let $\zeta \in\{1,2,3\}$ and suppose that for all $\beta \in \Omega$, for all $u \in U$, and for all $V \in 2^{U}$,

$$
\begin{aligned}
& J_{\beta}(u, V)=\inf \left\{J_{\beta}(u, w): w \in V\right\} \\
& \wedge J_{\beta}(V, u)=\inf \left\{J_{\beta}(w, u): w \in V\right\} .
\end{aligned}
$$

(a) Define on $C l^{L-\mathcal{J}_{s ; \Omega}}(U)\left(C l^{R-\mathcal{J}_{s ; \Omega}}(U)\right)$, the left (right) quasi-b-distance $\mathcal{D}_{\zeta}^{L-\mathcal{J}_{s ; \Omega}}=\left\{D_{\zeta ; \beta}^{L-\mathcal{J}_{s ; \Omega}}, \beta \in \Omega\right\}\left(\mathcal{D}_{\zeta}^{R-\mathcal{J}_{s ; \Omega}}=\left\{D_{\zeta ; \beta}^{R-\mathcal{J}_{s ; \Omega}}, \beta \in \Omega\right\}\right)$ of the Hausdorff type, where $D_{\zeta ; \beta}^{L-\mathcal{J}_{s ; \Omega}}: C l^{L-\mathcal{J}_{s ; \Omega}}(U) \times C l^{L-\mathcal{J}_{s ; \Omega}}(U) \rightarrow[0, \infty]$

$\left(D_{\zeta ; \beta}^{R-\mathcal{J}_{s ; \Omega}}: C l^{R-\mathcal{J}_{s ; \Omega}}(U) \times C l^{R-\mathcal{J}_{s ; \Omega}}(U) \rightarrow[0, \infty], \beta \in \Omega\right)$ for all $\beta \in \Omega$ and for all $U, V \in \mathrm{Cl}^{\mathcal{J}_{s ; \Omega} \Omega}(U)$ as:

$$
D_{1 ; \beta}^{L-\mathcal{J}_{s ; \Omega}}(U, V)=\max \left\{\sup _{u \in U} J_{\beta}(u, V), \sup _{v \in V} J_{\beta}(U, v)\right\},
$$




$$
\begin{aligned}
& D_{2 ; \beta}^{L-\mathcal{J}_{s ; \Omega}}(U, V)=\max \left\{\sup _{u \in U} J_{\beta}(u, V), \sup _{v \in V} J_{\beta}(v, U)\right\} \quad \text { and } \\
& D_{3 ; \beta}^{L-\mathcal{J}_{s ; \Omega}}(U, V)=\sup _{u \in U} J_{\beta}(u, V), \quad \text { if } \mathcal{J}_{s ; \Omega} \in \mathbb{J}_{(U, Q)}^{L} ;
\end{aligned}
$$

(a.2)

$$
\begin{aligned}
& D_{1 ; \beta}^{R-\mathcal{J}_{s ; \Omega}}(U, V)=\max \left\{\sup _{u \in U} J_{\beta}(u, V), \sup _{v \in V} J_{\beta}\right\}(U, v), \\
& D_{2 ; \beta}^{R-\mathcal{J}_{s ; \Omega}}(U, V)=\max \left\{\sup _{u \in U} J_{\beta}(u, V), \sup _{v \in V} J_{\beta}(v, U)\right\} \text { and } \\
& D_{3 ; \beta}^{R-\mathcal{J}_{s ; \Omega}}(U, V)=\sup _{u \in U} J_{\beta}(u, V), \quad \text { if } \mathcal{J}_{s ; \Omega} \in \mathbb{J}_{(U, Q)}^{R} .
\end{aligned}
$$

(b) Let $\mu=\left\{\mu_{\beta}\right\}_{\beta \in \Omega} \in[0,1)^{\Omega}$. The set-valued map $T: U \rightarrow C l^{L-\mathcal{J}_{s ; \Omega}}(U)$ $\left(T: U \rightarrow C l^{R-\mathcal{J}_{s ; \Omega}}(U)\right)$ is a left (right) $\left(\mathcal{D}_{\zeta}^{L-\mathcal{J}_{s ; \Omega}}, \mu\right)$-contraction on $U$ $\left(\left(\mathcal{D}_{\zeta}^{R-\mathcal{J}_{s ; \Omega}}, \mu\right)\right.$-contraction on $\left.U\right)$ if for all $\beta \in \Omega$ and for all $x, y \in U$ :

(b.1) $D_{\zeta ; \beta}^{L-\mathcal{J}_{s ; \Omega}}(T(x), T(y)) \leq \mu_{\beta} J_{\beta}(x, y)$, if $\mathcal{J}_{s ; \Omega} \in \mathbb{J}_{(U, Q)}^{L}$;

(b.2) $D_{\zeta ; \beta}^{R-\mathcal{J}_{s ; \Omega}}(T(x), T(y)) \leq \mu_{\beta} J_{\beta}(x, y)$, if $\mathcal{J}_{s ; \Omega} \in \mathbb{J}_{(U, Q)}^{R}$.

Remark 3.21 Let $\left(U, Q_{s ; \Omega}\right)$ be a quasi-b-gauge space, and let $\mathcal{J}_{s ; \Omega}=\left\{J_{\beta}: \beta \in \Omega\right\}$ of maps $J_{\beta}: U \times U \rightarrow[0, \infty), \beta \in \Omega$ be a left (right) $\mathcal{J}_{s ; \Omega}$-family on $U$.

(a) In general, $D_{\zeta ; \beta}^{L-\mathcal{J}_{s ; \Omega}}\left(D_{\zeta ; \beta}^{R-\mathcal{J}_{s ; \Omega}}\right)$ are not symmetric, thus $D_{\zeta ; \beta}^{L-\mathcal{J}_{s ; \Omega}}(U, V)=D_{\zeta ; \beta}^{L-\mathcal{J}_{s ; \Omega}}(V, U)\left(D_{\zeta ; \beta}^{R-\mathcal{J}_{s ; \Omega}}(U, V)=D_{\zeta ; \beta}^{R-\mathcal{J}_{s ; \Omega}}(V, U)\right)$ does not necessarily hold. Also, $D_{\zeta ; \beta}^{L-\mathcal{J}_{s ; \Omega}}(U, U)=0\left(D_{\zeta ; \beta}^{R-\mathcal{J}_{s ; \Omega}}(U, U)=0\right)$ does not necessarily hold (See Remark 3.27 (b) and (c) for details).

(b) Each $\left(\mathcal{D}_{\zeta}^{L-\mathcal{J}_{s ; \Omega}}, \mu\right)$-contraction on $U\left(\left(\mathcal{D}_{\zeta}^{R-\mathcal{J}_{s ; \Omega}}, \mu\right)\right.$-contraction on $\left.U\right), \zeta \in\{1,2,3\}$ is $\left(\mathcal{D}_{3}^{L-\mathcal{J}_{s ; \Omega}}, \mu\right)$-contraction on $U\left(\left(\mathcal{D}_{3}^{R-\mathcal{J}_{s ; \Omega}}, \mu\right)\right.$-contraction on $\left.U\right)$, but the converse is not generally true.

Our main result for set-valued mappings is given below.

Theorem 3.22 Let $\left(U, Q_{s ; \Omega}\right)$ be a quasi-b-gauge space. Let $\mathcal{J}_{s ; \Omega}=\left\{J_{\beta}: \beta \in \Omega\right\}$ be a left (right) $\mathcal{J}_{s ; \Omega}$-family on $U$ and let $\zeta \in\{1,2,3\}$. Assume, moreover, that $\mu=\left\{\mu_{\beta}\right\}_{\beta \in \Omega} \in[0,1)$, and the set-valued map $T: U \rightarrow C l^{L-\mathcal{J}_{s ; \Omega}}(U)\left(T: U \rightarrow C l^{R-\mathcal{J}_{s ; \Omega}}(U)\right)$ satisfies:

(i) $T$ is a $\left(\mathcal{D}_{\zeta}^{L-\mathcal{J}_{s ; \Omega}}, \mu\right)$-contraction on $U\left(\left(\mathcal{D}_{\zeta}^{R-\mathcal{J}_{s ; \Omega}}, \mu\right)\right.$-contraction on $\left.U\right)$;

(ii) for any $u \in U$ and any $\gamma=\left\{\gamma_{\beta}\right\}_{\beta \in \Omega} \in(0, \infty)$, there exists $v \in T(u)$ such that for all $\beta \in \Omega$,

$$
\begin{aligned}
& J_{\beta}(u, v)<J_{\beta}(u, T(u))+\gamma_{\beta}, \\
& \left(J_{\beta}(v, u)<J_{\beta}(T(u), u)+\gamma_{\beta}\right) .
\end{aligned}
$$

We have the following:

(I) If $(U, T)$ at a point $z^{0} \in U$ is the left (right) $\mathcal{J}_{s ; \Omega}$-admissible, then there exists a sequence $\left\{z^{m}: m \in\{0\} \cup \mathbb{N}\right\}$ starting at $z^{0} \in U$ such that $z^{m} \in T\left(z^{m-1}\right)$ for all $m \in \mathbb{N}$, a point $z \in U$ and $r=\left\{r_{\beta}\right\}_{\beta \in \Omega} \in(0, \infty)$ such that $z^{m} \in B^{L-\mathcal{J}_{s ; \Omega}}\left(z^{0}, r\right)$ $\left.\left(z^{m} \in B^{R-\mathcal{J}_{s ; \Omega}}\left(z^{0}, r\right)\right\}\right)$ for all $m \in \mathbb{N}$ and $\lim _{m \rightarrow \infty}^{L-\mathcal{J}_{s ; \Omega}} z_{m}=z\left(\lim _{m \rightarrow \infty}^{R-\mathcal{J}_{s ; \Omega}} z_{m}=z\right)$. 
(II) If $(U, T)$ at a point $z^{0} \in U$ is the left (right) $\mathcal{J}_{s ; \Omega}$-admissible and if $T^{[k]}$, for some $k \in \mathbb{N}$, is a left (right) $Q_{s ; \Omega}$-quasi-closed map on $U$, then $\operatorname{Fix}\left(T^{[k]}\right)$ is non-empty and there exists a sequence $\left\{z^{m}: m \in\{0\} \cup \mathbb{N}\right\}$ starting at $z^{0} \in U$ such that $z^{m} \in T\left(z^{m-1}\right)$ for all $m \in \mathbb{N}$, a point $z \in \operatorname{Fix}\left(T^{[k]}\right)$ and $r=\left\{r_{\beta}\right\}_{\beta \in \Omega} \in(0, \infty)$ such that $z^{m} \in B^{L-\mathcal{J}_{s ; \Omega}}\left(z^{0}, r\right)\left(z^{m} \in B^{R-\mathcal{J}_{s ; \Omega}}\left(z^{0}, r\right)\right)$ for all $m \in \mathbb{N}$ and $\lim _{m \rightarrow \infty}^{L-\mathcal{J}_{s ; \Omega}} z_{m}=z$ $\left(\lim _{m \rightarrow \infty}^{R-\mathcal{J}_{s ; \Omega}} z_{m}=z\right)$.

Proof (I) Suppose that $(U, T)$ is the left (right) $\mathcal{J}_{s ; \Omega}$-admissible at a point $z^{0} \in U$.

From using (3.14) and the fact that $J_{\beta}: U \times U \rightarrow[0, \infty), \beta \in \Omega$, we choose

$$
\begin{aligned}
& r=\left\{r_{\beta}\right\}_{\beta \in \Omega} \in(0, \infty), \\
& s=\left\{s_{\beta}\right\}_{\beta \in \Omega} \in[1, \infty)
\end{aligned}
$$

such that for all $\beta \in \Omega$

$$
J_{\beta}\left(z^{0}, T\left(z^{0}\right)\right)<\frac{\left(1-\mu_{\beta}\right) r_{\beta}}{s_{\beta}} .
$$

Put

$$
\gamma_{\beta}^{(0)}=\frac{\left(1-\mu_{\beta}\right) r_{\beta}}{s_{\beta}}-J_{\beta}\left(z^{0}, T\left(z^{0}\right)\right) \quad \text { for all } \beta \in \Omega
$$

From (3.17), (3.18), and (3.19), we have $\gamma^{(0)}=\left\{\gamma_{\beta}^{(0)}\right\}_{\beta \in \Omega} \in(0, \infty)$. Applying (3.15), we get $z^{1} \in T\left(z^{(0)}\right)$ such that

$$
J_{\beta}\left(z^{0}, z^{1}\right)<J_{\beta}\left(z^{0}, T\left(z^{0}\right)\right)+\gamma_{\beta}^{(0)} \quad \text { for all } \beta \in \Omega
$$

We see from (3.20) and (3.21) that

$$
J_{\beta}\left(z^{0}, z^{1}\right)<\frac{\left(1-\mu_{\beta}\right) r_{\beta}}{s_{\beta}} \quad \text { for all } \beta \in \Omega
$$

Observe that (3.22) implies $z^{1} \in B^{L-\mathcal{J}_{s ; \Omega}}\left(z^{0}, r\right)$.

\section{Put now}

$$
\gamma_{\beta}^{(1)}=\mu_{\beta}\left[\frac{\left(1-\mu_{\beta}\right) r_{\beta}}{s_{\beta}^{2}}-J_{\beta}\left(z^{0}, z^{1}\right)\right] \text { for all } \beta \in \Omega
$$

From (3.22), we have $\gamma^{(1)}=\left\{\gamma_{\beta}^{(1)}\right\}_{\beta \in \Omega} \in(0, \infty)$, and we apply (3.15) to find $z^{2} \in T\left(z^{(1)}\right)$ such that

$$
J_{\beta}\left(z^{1}, z^{2}\right)<J_{\beta}\left(z^{1}, T\left(z^{1}\right)\right)+\gamma_{\beta}^{(1)} \quad \text { for all } \beta \in \Omega \text {. }
$$

Also, note that

$$
J_{\beta}\left(z^{1}, z^{2}\right)<\frac{\mu_{\beta}\left(1-\mu_{\beta}\right) r_{\beta}}{s_{\beta}^{2}} \quad \text { for all } \beta \in \Omega
$$


Indeed, from (3.24), (3.14), Definition 3.20, and (3.23), we get for all $\beta \in \Omega$,

$$
\begin{aligned}
J_{\beta}\left(z^{1}, z^{2}\right) & <J_{\beta}\left(z^{1}, T\left(z^{1}\right)\right)+\gamma_{\beta}^{(1)} \\
& \leq \sup _{u \in T\left(z^{0}\right)} J_{\beta}\left(u, T\left(z^{1}\right)\right)+\gamma_{\beta}^{(1)} \\
& \leq D_{\zeta ; \beta}^{L-\mathcal{J}_{s ; \Omega}}\left(T\left(z^{0}\right), T\left(z^{1}\right)\right)+\gamma_{\beta}^{(1)} \\
& \leq \mu_{\beta} J_{\beta}\left(z^{0}, z^{1}\right)+\gamma_{\beta}^{(1)}=\frac{\mu_{\beta}\left(1-\mu_{\beta}\right) r_{\beta}}{s_{\beta}^{2}}, \quad \zeta \in\{1,2,3\} .
\end{aligned}
$$

Thus, (3.25) holds. Further, by $\left(\mathcal{J}_{1}\right)$ there exists $s=\left\{s_{\beta}\right\}_{\beta \in \Omega} \in[1, \infty)$. Using (3.22) and (3.25), we have for all $\beta \in \Omega$,

$$
\begin{aligned}
J_{\beta}\left(z^{0}, z^{2}\right) & \leq s_{\beta}\left\{J_{\beta}\left(z^{0}, z^{1}\right)+J_{\beta}\left(z^{1}, z^{2}\right)\right\} \\
& <s_{\beta}\left\{\frac{\left(1-\mu_{\beta}\right) r_{\beta}}{s_{\beta}}+\frac{\mu_{\beta}\left(1-\mu_{\beta}\right) r_{\beta}}{s_{\beta}^{2}}\right\} \\
& \leq\left(1-\mu_{\beta}\right) r_{\beta}\left(1+\frac{\mu_{\beta}}{s_{\beta}}\right) \leq\left(1-\mu_{\beta}\right) r_{\beta}\left(1+\mu_{\beta}\right) \\
& \leq\left(1-\mu_{\beta}\right) r_{\beta} \sum_{k=0}^{\infty} \mu_{\beta}^{k}=r_{\beta} .
\end{aligned}
$$

Thus, $z^{2} \in B^{L-\mathcal{J}_{s ; \Omega}}\left(z^{0}, r\right)$. Repeating the above process, using Definition 3.20 and property (3.15), we find a sequence $\left\{z^{m}\right\}_{m \in \mathbb{N}}$ in $U$ satisfying

$$
z^{m+1} \in T\left(z^{m}\right) \quad \text { for all } m \in\{0\} \cup \mathbb{N} \text {. }
$$

Letting $\gamma^{(m)}=\left\{\gamma_{\beta}^{(m)}\right\}_{\beta \in \Omega}$ for all $m \in \mathbb{N}$, where

$$
\gamma_{\beta}^{(m)}=\mu_{\beta}\left[\frac{\mu_{\beta}^{m-1}\left(1-\mu_{\beta}\right) r_{\beta}}{s_{\beta}^{m+1}}-J_{\beta}\left(z^{m-1}, z^{m}\right)\right] .
$$

We also notice that $\left\{\gamma^{(m)} \in(0, \infty): m \in \mathbb{N}\right\}$ and for all $\beta \in \Omega$ and for all $m \in\{0\} \cup \mathbb{N}$, we have

$$
\begin{aligned}
& J_{\beta}\left(z^{m}, z^{m+1}\right)<J_{\beta}\left(z^{m}, T\left(z^{m}\right)\right)+\gamma^{(m)}, \\
& J_{\beta}\left(z^{m}, z^{m+1}\right)<\frac{\mu_{\beta}^{m}\left(1-\mu_{\beta}\right) r_{\beta}}{s_{\beta}^{m+1}} .
\end{aligned}
$$

For all $\beta \in \Omega$ and for all $m \in\{0\} \cup \mathbb{N}$, we can write

$$
\begin{aligned}
J_{\beta}\left(z^{0}, z^{m+1}\right) \leq & s_{\beta} J_{\beta}\left(z^{0}, z^{1}\right)+s_{\beta}^{2} J_{\beta}\left(z^{1}, z^{2}\right)+s_{\beta}^{3} J_{\beta}\left(z^{2}, z^{3}\right) \\
& +\cdots+s_{\beta}^{m} J_{\beta}\left(z^{m-1}, z^{m}\right)+s_{\beta}^{m} J_{\beta}\left(z^{m}, z^{m+1}\right) \\
< & s_{\beta} \frac{\left(1-\mu_{\beta}\right) r_{\beta}}{s_{\beta}}+s_{\beta}^{2} \frac{\mu_{\beta}\left(1-\mu_{\beta}\right) r_{\beta}}{s_{\beta}^{2}}+s_{\beta}^{3} \frac{\mu_{\beta}^{2}\left(1-\mu_{\beta}\right) r_{\beta}}{s_{\beta}^{3}}
\end{aligned}
$$




$$
\begin{aligned}
& +\cdots+s_{\beta}^{m} \frac{\mu_{\beta}^{m-1}\left(1-\mu_{\beta}\right) r_{\beta}}{s_{\beta}^{m}}+s_{\beta}^{m} \frac{\mu_{\beta}^{m}\left(1-\mu_{\beta}\right) r_{\beta}}{s_{\beta}^{m+1}} \\
= & \left(1-\mu_{\beta}\right) r_{\beta}\left\{1+\mu_{\beta}+\mu_{\beta}^{2} \cdots \mu_{\beta}^{m-1}+\frac{\mu_{\beta}^{m}}{s_{\beta}}\right\} \\
\leq & \left(1-\mu_{\beta}\right) r_{\beta}\left\{1+\mu_{\beta}+\mu_{\beta}^{2} \cdots \mu_{\beta}^{m-1}+\mu_{\beta}^{m}\right\} \\
= & \left(1-\mu_{\beta}\right) r_{\beta} \sum_{k=0}^{m} \mu_{\beta}^{k} . \\
< & \left(1-\mu_{\beta}\right) r_{\beta} \sum_{k=0}^{\infty} \mu_{\beta}^{k}=r_{\beta} .
\end{aligned}
$$

Hence, this implies that $z^{m} \in B^{L-\mathcal{J}_{s ; \Omega}}\left(z^{0}, r\right)$ for all $m \in \mathbb{N}$.

Using (J1) and (3.27), for all $m, n \in \mathbb{N}$ such that $n>m$, we have

$$
\begin{aligned}
\lim _{m \rightarrow \infty} \sup _{n>m} J_{\beta}\left(z^{m}, z^{n}\right) \leq & \lim _{m \rightarrow \infty} \sup _{n>m}\left\{s_{\beta} J_{\beta}\left(z^{m}, z^{m+1}\right)+s_{\beta}^{2} J_{\beta}\left(z^{m+1}, z^{m+2}\right)\right. \\
& \left.+\cdots+s_{\beta}^{n-m-1} J_{\beta}\left(z^{n-2}, z^{n-1}\right)+s_{\beta}^{n-m-1} J_{\beta}\left(z^{n-1}, z^{n}\right)\right\} \\
\leq & \lim _{m \rightarrow \infty} \sup _{n>m}\left\{s_{\beta} \frac{\mu_{\beta}^{m}\left(1-\mu_{\beta}\right) r_{\beta}}{s_{\beta}^{m+1}}+s_{\beta}^{2} \frac{\mu_{\beta}^{m+1}\left(1-\mu_{\beta}\right) r_{\beta}}{s_{\beta}^{m+2}}\right. \\
& \left.+\cdots+s_{\beta}^{n-m-1} \frac{\mu_{\beta}^{n-2}\left(1-\mu_{\beta}\right) r_{\beta}}{s_{\beta}^{n-1}}+s_{\beta}^{n-m-1} \frac{\mu_{\beta}^{n-1}\left(1-\mu_{\beta}\right) r_{\beta}}{s_{\beta}^{n-1+1}}\right\} \\
\leq & \lim _{m \rightarrow \infty} \sup _{n>m}\left(1-\mu_{\beta}\right) r_{\beta}\left\{\frac{\mu_{\beta}^{m}}{s_{\beta}^{m}}+\frac{\mu_{\beta}^{m+1}}{s_{\beta}^{m}}+\cdots+\frac{\mu_{\beta}^{n-2}}{s_{\beta}^{m}}+\frac{\mu_{\beta}^{n-1}}{s_{\beta}^{m+1}}\right\} \\
\leq & \lim _{m \rightarrow \infty} \sup _{n>m}\left(1-\mu_{\beta}\right) r_{\beta}\left\{\mu_{\beta}^{m}+\mu_{\beta}^{m+1}+\cdots+\mu_{\beta}^{n-2}+\mu_{\beta}^{n-1}\right\} \\
= & \left(1-\mu_{\beta}\right) r_{\beta} \lim _{m \rightarrow \infty} \sup _{n>m} \sum_{j=m}^{n-1} \mu_{\beta}^{j} \\
\leq & r_{\beta} \lim _{m \rightarrow \infty} \mu_{\beta}^{m} .
\end{aligned}
$$

This implies

$$
\forall \beta \in \Omega\left\{\lim _{m \rightarrow \infty} \sup _{n>m} J_{\beta}\left(z^{m}, z^{n}\right)=0\right\} \quad \text { for all } \beta \in \Omega .
$$

Given that $(U, T)$ is thr left $\mathcal{J}_{s ; \Omega}$-admissible on $U$, hence using Definition 3.16, properties (3.26) and (3.28), we find $z \in U$ such that

$$
\lim _{m \rightarrow \infty} J_{\beta}\left(z, z^{m}\right)=0 \quad \text { for all } \beta \in \Omega .
$$

Now, taking $v_{m}=z$ and $u_{m}=z^{m}$ for $m \in \mathbb{N}$, we observe that condition (3.4) and (3.6) hold for $\left\{u_{m}\right\}$ and $\left\{v_{m}\right\}$ in $U$ by (3.28) and (3.29). Consequently, we get (3.8) by $(\mathcal{J} 2)$ which gives

$$
\lim _{m \rightarrow \infty} q_{\beta}\left(z, z^{m}\right)=\lim _{m \rightarrow \infty} q_{\beta}\left(v_{m}, u_{m}\right)=0 \quad \text { for all } \beta \in \Omega
$$

Thus, we have $z \in S_{\left(z_{m}: m \in \mathbb{N}\right)}^{L-Q_{s ; \Omega}}=\left\{x \in U: \lim _{m \rightarrow \infty}^{L-Q_{s ; \Omega}} z^{m}=x\right\}$. 
(II) Let $(U, T)$ be the left $\mathcal{J}_{s ; \Omega}$-admissible at a point $z^{0} \in U$ and $T^{[k]}$ be the left $Q_{s ; \Omega}$-quasiclosed on $U$, for some $k \in \mathbb{N}$.

Let $z^{0} \in U$ be arbitrary. Since $S_{\left(z^{m}: m \in\{0\} \cup \mathbb{N}\right)}^{L-Q_{s ; \Omega}} \neq \emptyset$ and for $m \in\{0\} \cup \mathbb{N}$, we have

$$
z^{(m+1) k} \in T^{[k]}\left(z^{m k}\right)
$$

By defining $\left\{z_{m}=z^{m-1+k}: m \in \mathbb{N}\right\}$, we can write

$$
z_{m} \subset T^{[k]}(U)
$$

and

$$
S_{\left(z_{m}: m \in\{0\} \cup \mathbb{N}\right)}^{L-Q_{s ;}}=S_{\left(z^{m}: m \in\{0\} \cup \mathbb{N}\right)}^{L-Q_{s}} \neq \emptyset .
$$

Also, its subsequences

$$
\left\{y_{m}=z^{(m+1) k}: m \in \mathbb{N}\right\} \subset T^{[k]}(U)
$$

and

$$
\left\{x_{m}=z^{m k}: m \in \mathbb{N}\right\} \subset T^{[k]}(U)
$$

satisfy

$$
y_{m}=T^{[k]}\left(x_{m}\right) \quad \text { for all } m \in \mathbb{N}
$$

and are the left $Q_{s ; \Omega^{-}}$convergent to each point $z \in S_{\left(z^{m}: m \in\{0\} \cup \mathbb{N}\right)}^{L-Q_{s ; \Omega}}$. Now, since

$$
S_{\left(z_{m}: m \in \mathbb{N}\right)}^{L-Q_{s ; \Omega}} \subset S_{\left(y_{m}: m \in \mathbb{N}\right)}^{L-Q_{s ; \Omega}} \quad \text { and } \quad S_{\left(z_{m}: m \in \mathbb{N}\right)}^{L-Q_{s ; \Omega}} \subset S_{\left(x_{m}: m \in \mathbb{N}\right)}^{L-Q_{s ; \Omega}},
$$

using the assumption that $T^{[k]}$ for some $k \in \mathbb{N}$ is a left (right) $Q_{s ; \Omega}$-quasi-closed map on $U$, there exists $z \in S_{\left(z_{m}: m \in\{0\} \cup \mathbb{N}\right)}^{L-Q_{s ;}}=S_{\left(z^{m}: m \in\{0\} \cup \mathbb{N}\right)}^{L-Q_{s ; \Omega}}$ such that $z \in T^{[k]}(z)$. This completes the proof.

We now extend the above theorems to the Banach type single-valued left (right)contractions.

Definition 3.23 Let $\left(U, Q_{s ; \Omega}\right)$ be a quasi- $b$-gauge space, let $\mathcal{J}_{s ; \Omega}=\left\{J_{\beta}: \beta \in \Omega\right\}$ is a left (right) $\mathcal{J}_{s ; \Omega}$-family on $U$, and let $\zeta \in\{1,2\}$.

(c) The left (right) $b$-distance $\mathcal{D}_{\zeta}^{L-\mathcal{J}_{s ; \Omega}}=\left\{D_{\zeta ; \beta}^{L-\mathcal{J}_{s ; \Omega}}, \beta \in \Omega\right\}\left(\mathcal{D}_{\zeta}^{R-\mathcal{J}_{s ; \Omega}}=\left\{D_{\zeta ; \beta}^{R-\mathcal{J}_{s ; \Omega}}, \beta \in \Omega\right\}\right)$ on $U$, where $D_{\zeta ; \beta}^{L-\mathcal{J}_{s ; \Omega}}: U \times U \rightarrow[0, \infty), \beta \in \Omega\left(D_{\zeta ; \beta}^{R-\mathcal{J}_{s ; \Omega}}: U \times U \rightarrow[0, \infty), \beta \in \Omega\right)$ are defined for all $\beta \in \Omega$ and for all $u, v \in U$ as follows:

(c.1)

$$
\begin{aligned}
& D_{1 ; \beta}^{L-\mathcal{J}_{s ; \Omega}}(u, v)=\max \left\{J_{\beta}(u, v), J_{\beta}(v, u)\right\} \\
& D_{2 ; \beta}^{L-\mathcal{J}_{s ; \Omega}}(u, v)=J_{\beta}(u, v), \quad \text { if } \mathcal{J}_{s ; \Omega} \in \mathbb{J}_{\left(U, Q_{s ; \Omega}\right)}^{L} ;
\end{aligned}
$$


(c.2)

$$
\begin{aligned}
& D_{1 ; \beta}^{R-\mathcal{J}_{s ; \Omega}}(u, v)=\max \left\{J_{\beta}(u, v), J_{\beta}(v, u)\right\}, \\
& D_{2 ; \beta}^{R-\mathcal{J}_{s ; \Omega}}(u, v)=J_{\beta}(u, v), \quad \text { if } \mathcal{J}_{s ; \Omega} \in \mathbb{J}_{\left(U, Q_{s ; \Omega}\right)}^{R} .
\end{aligned}
$$

(d) Let $\mu=\left\{\mu_{\beta}\right\}_{\beta \in \Omega} \in[0,1)$. A single-valued map $T: U \rightarrow U$ is $\left(\mathcal{D}_{\zeta}^{L-\mathcal{J}_{s ; \Omega}}, \mu\right)$-contraction on $U\left(\left(\mathcal{D}_{\zeta}^{R-\mathcal{J}_{s ; \Omega},}, \mu\right)\right.$-contraction on $\left.U\right)$ if for all $\beta \in \Omega$ and for all $x, y \in U$ :

(d.1) $D_{\zeta ; \beta}^{L-\mathcal{J}_{s ; \Omega}}(T(x), T(y)) \leq \mu_{\beta} J_{\beta}(x, y)$, if $\mathcal{J}_{s ; \Omega} \in \mathbb{J}_{\left(U, Q_{s ; \Omega}\right)}^{L}$;

(d.2) $D_{\zeta ; \beta}^{R-\mathcal{J}_{s ; \Omega}}(T(x), T(y)) \leq \mu_{\beta} J_{\beta}(x, y)$, if $\mathcal{J}_{s ; \Omega} \in \mathbb{J}_{\left(U, Q_{s ; \Omega}\right)}^{R}$.

As a result of Definition 3.23 and Theorem 3.22, we now have the following theorem.

Theorem 3.24 Let $\left(U, Q_{s ; \Omega}\right)$ be a quasi-b-gauge space. Let $\mathcal{J}_{s ; \Omega}=\left\{J_{\beta}: \beta \in \Omega\right\}$ be a left (right) $\mathcal{J}_{s ; \Omega}$-family of generalized quasi-pseudo-b-distances on $U$, and let $\zeta \in\{1,2\}$. Moreover, assume that $\mu=\left\{\mu_{\beta}\right\}_{\beta \in \Omega} \in[0,1)$ and $T: U \rightarrow U$ is a $\left(\mathcal{D}_{\zeta}^{L-\mathcal{J}_{s ; \Omega}}, \mu\right)$-contraction on $U$ $\left(\left(\mathcal{D}_{\zeta}^{R-\mathcal{J}_{s ; \Omega}}, \mu\right)\right.$-contraction on $\left.U\right)$.

(I) If $(U, T)$ is the left (right) $\mathcal{J}_{s ; \Omega}$-admissible at a point $z^{0} \in U$, then there is a sequence $\left\{z^{m}: m \in\{0\} \cup \mathbb{N}\right\}$ starting at $z^{0} \in U$ with $\left\{z^{m}=T^{[m]}\left(z^{0}\right): m \in\{0\} \cup \mathbb{N}\right\}$, a point $z \in U$, and $r=\left\{r_{\beta}\right\}_{\beta \in \Omega} \in(0, \infty)$ such that $z^{m} \in B^{L-\mathcal{J}_{s ; \Omega}}\left(z^{0}, r\right)\left(z^{m} \in B^{R-\mathcal{J}_{s ; \Omega}}\left(z^{0}, r\right)\right)$ for all $m \in \mathbb{N}$ and $\lim _{m \rightarrow \infty}^{L-\mathcal{J}_{s ; \Omega}} z_{m}=z\left(\lim _{m \rightarrow \infty}^{R-\mathcal{J}_{s ; \Omega}} z_{m}=z\right)$.

(II) If $(U, T)$ is the left (right) $\mathcal{J}_{s ; \Omega}$-admissible at a point $z^{0} \in U$ and if $T^{[k]}$ for some $k \in \mathbb{N}$ is a left (right) $Q_{s ; \Omega}$-quasi-closed map on $U$, then $\operatorname{Fix}\left(T^{[k]}\right)$ is non-empty, and there exists a sequence $\left\{z^{m}: m \in 0 \cup \mathbb{N}\right\}$ starting at $z^{0} \in U$ with $\left\{z^{m}=T^{[m]}\left(z^{0}\right): m \in\{0\} \cup \mathbb{N}\right\}$, a point $z \in \operatorname{Fix}\left(T^{[k]}\right)$, and $r=\left\{r_{\beta}\right\}_{\beta \in \Omega} \in(0, \infty)$ such that $z^{m} \in B^{L-\mathcal{J}_{s ; \Omega}}\left(z^{0}, r\right)\left(z^{m} \in B^{R-\mathcal{J}_{s ; \Omega}}\left(z^{0}, r\right)\right)$ for all $m \in \mathbb{N}, \lim _{m \rightarrow \infty}^{L-\mathcal{J}_{s, \Omega}} z_{m}=z$ $\left(\lim _{m \rightarrow \infty}^{R-\mathcal{J}_{s, \Omega}} z_{m}=z\right)$, and we have

$$
J_{\beta}(z, T(z))=J_{\beta}(T(z), z)=0, \quad \text { for all } \beta \in \Omega \text { and for all } z \in \operatorname{Fix}\left(T^{[k]}\right)
$$

(III) If $\left(U, Q_{s ; \Omega}\right)$ is a Hausdorff space, and if $(U, T)$ at a point $z^{0} \in U$ is lthe eft (right) $\mathcal{J}_{s ; \Omega}$-admissible, and if $T^{[k]}$, for some $k \in \mathbb{N}$, is a left (right) $Q_{s ; \Omega}$-quasi-closed map on $U$, then there exists a sequence $\left\{z^{m}: m \in 0 \cup \mathbb{N}\right\}$ starting at $z^{0} \in U$ with $\left\{z^{m}=T^{[m]}\left(z^{0}\right): m \in\{0\} \cup \mathbb{N}\right\}$, a point $z \in \operatorname{Fix}\left(T^{[k]}\right)=\operatorname{Fix}(T)=\{z\}$ and $r=\left\{r_{\beta}\right\}_{\beta \in \Omega} \in(0, \infty)$ such that $z^{m} \in B^{L-\mathcal{J}_{s ; \Omega}}\left(z^{0}, r\right)\left(z^{m} \in B^{R-\mathcal{J}_{s ; \Omega}}\left(z^{0}, r\right)\right)$ for all $m \in \mathbb{N}$, $\lim _{m \rightarrow \infty}^{L-\mathcal{J}_{s ; \Omega}} z_{m}=z\left(\lim _{m \rightarrow \infty}^{R-\mathcal{J}_{s ; \Omega}} z_{m}=z\right)$, and we have

$$
J_{\beta}(z, z)=0 \quad \text { for all } \beta \in \Omega
$$

Proof We prove only (3.30) and (3.31).

On contrary, suppose that there exist $\beta_{0} \in \Omega$ and $z \in \operatorname{Fix}\left(T^{[k]}\right)$ such that $J_{\beta_{0}}(z, T(z))>0$. Indeed, $z=T^{[2 k]}(z), T(z)=T^{[2 k]}(T(z))$ and for $\zeta \in\{1,2\}$, by Definition (3.23),

$$
\begin{aligned}
0 & <J_{\beta_{0}}(z, T(z))=J_{\beta_{0}}\left(T^{[2 k]}(z), T^{[2 k]}(T(z))\right) \\
& \leq D_{\zeta ; \beta_{0}}^{L-\mathcal{J}_{s ; \Omega}}\left(T^{[2 k]}(z), T^{[2 k]}(T(z))\right)
\end{aligned}
$$




$$
\begin{aligned}
& \leq \mu_{\beta_{0}} J_{\beta_{0}}\left(T^{[2 k-1]}(z), T^{[2 k-1]}(T(z))\right) \\
& \leq \mu_{\beta_{0}} D_{\zeta ; \beta_{0}}^{L-\mathcal{J}_{s ; \Omega}}\left(T^{[2 k-1]}(z), T^{[2 k-1]}(T(z))\right) \\
& \leq \mu_{\beta_{0}}^{2} J_{\beta_{0}}\left(T^{[2 k-2]}(z), T^{[2 k-2]}(T(z))\right) \leq \cdots \\
& \leq \mu_{\beta_{0}}^{2 k} J_{\beta_{0}}(z, T(z))<J_{\beta_{0}}(z, T(z)),
\end{aligned}
$$

which is a contradiction.

Now, suppose that there exist $\beta_{0} \in \Omega$ and $z \in \operatorname{Fix}\left(T^{[k]}\right)$ such that $J_{\beta_{0}}(T(z), z)>0$. Then, Definition 3.23 and the fact that $z=T^{[k]}(z)=T^{[2 k]}(z)$ imply that for $\zeta \in\{1,2\}$,

$$
\begin{aligned}
0< & J_{\beta_{0}}(T(z), z)=J_{\beta_{0}}\left(T^{[k+1]}(z), T^{[2 k]}(z)\right) \\
\leq & s_{\beta_{0}} J_{\beta_{0}}\left(T^{[k+1]}(z), T^{[k+2]}(z)\right)+s_{\beta_{0}}^{2} J_{\beta_{0}}\left(T^{[k+2]}(z), T^{[k+3]}(z)\right) \\
& +\cdots+s_{\beta_{0}}^{k-2} J_{\beta_{0}}\left(T^{[2 k-1]}(z), T^{[2 k]}(z)\right) \\
\leq & s_{\beta_{0}} D_{\zeta ; \beta_{0}}^{L-\mathcal{J}_{s ; \Omega}}\left(T^{[k+1]}(z), T^{[k+2]}(z)\right)+s_{\beta_{0}}^{2} D_{\zeta ; \beta_{0}}^{L-\mathcal{J}_{s ; \Omega}}\left(T^{[k+2]}(z), T^{[k+3]}(z)\right) \\
& +\cdots+s_{\beta_{0}}^{k-2} D_{\zeta ; \beta_{0}}^{L-\mathcal{J}_{s ; \Omega}}\left(T^{[2 k-1]}(z), T^{[2 k]}(z)\right) \\
\leq & s_{\beta_{0}} \mu_{\beta_{0}}^{k+1} J_{\beta_{0}}(z, T(z))+s_{\beta_{0}}^{2} \mu_{\beta_{0}}^{k+2} J_{\beta_{0}}(z, T(z)) \\
& +\cdots+s_{\beta_{0}}^{k-2} \mu_{\beta_{0}}^{2 k-1} J_{\beta_{0}}(z, T(z))=0,
\end{aligned}
$$

which is impossible. Thus, property (3.30) holds.

Next, we show that property (3.31) holds.

If the space $\left(U, Q_{s ; \Omega}\right)$ is the Hausdorff one, then Proposition (3.11) and property (3.30) suggest that $T(z)=z$ for all $z \in \operatorname{Fix}\left(T^{[k]}\right)$ and $J_{\beta}(z, z) \leq s_{\beta} J_{\beta}(z, T(z))+s_{\beta} J_{\beta}(T(z), z)=0$, for all $\beta \in \Omega$ and for all $z \in \operatorname{Fix}\left(T^{[k]}\right)$. Thus, $\operatorname{Fix}\left(T^{[k]}\right)=\operatorname{Fix}(T)$ and for all $z \in \operatorname{Fix}\left(T^{[k]}\right)=\operatorname{Fix}(T)$, we have $J_{\beta}(z, z)=0$.

To prove $\operatorname{Fix}(T)$ is a singleton, on the contrary, let $y, z \in \operatorname{Fix}(T)$ and $y \neq z$. Then, Proposition (3.11) implies there exists $\beta_{0} \in \Omega$ such that $J_{\beta_{0}}(y, z)>0 \vee J_{\beta_{0}}(z, y)>0$. Obviously, for $\zeta \in\{1,2\}$, we then have

$$
\begin{aligned}
& {\left[J_{\beta_{0}}(y, z)>0 \wedge J_{\beta_{0}}(y, z)=J_{\beta_{0}}(T(y), T(z)) \leq D_{\zeta ; \beta_{0}}^{L-\mathcal{J}_{s ; \Omega}}(T(y), T(z))\right.} \\
& \left.\quad \leq \mu_{\beta_{0}} J_{\beta_{0}}(y, z)<J_{\beta_{0}}(y, z)\right] \vee\left[J_{\beta_{0}}(z, y)>0 \wedge J_{\beta_{0}}(z, y)\right. \\
& \quad=J_{\beta_{0}}(T(z), T(y)) \leq D_{\zeta ; \beta_{0}}^{L-\mathcal{J}_{s ; \Omega}}(T(z), T(y)) \\
& \left.\quad \leq \mu_{\beta_{0}} J_{\beta_{0}}(z, y)<J_{\beta_{0}}(z, y)\right],
\end{aligned}
$$

which is impossible. Hence, we obtain $\operatorname{Fix}(T)=\{z\}$. The theorem is proved.

Remark 3.25 The proof of the right case in above theorems is based on an analogous technique.

Example 3.26 Let $U=[0,6]$, and let $Q_{s ; \Omega}=\{q\}$, where $q$ is a quasi-pseudo- $b$-metric on $U$ defined for all $u, v \in U$ by

$$
q(u, v)= \begin{cases}0 & \text { if } u \geq v \\ (u-v)^{2} & \text { if } u<v .\end{cases}
$$


Let $G=[0,3) \cup(3,6]$ be a subset of $U$. Let $\mathcal{J}_{s ; \Omega}=\{/\}$, where $J: U \times U \rightarrow[0, \infty)$ is defined for all $u, v \in U$ by

$$
J(u, v)= \begin{cases}q(u, v) & \text { if } G \cap\{u, v\}=\{u, v\}, \\ 40 & \text { if } G \cap\{u, v\} \neq\{u, v\} .\end{cases}
$$

The set-valued map $T$ is defined by

$$
T(u)= \begin{cases}{[4 ; 6]} & \text { for } u \in[0,3) \cup(3,6] \\ {[5,6]} & \text { for } u=3 .\end{cases}
$$

(I.1) $\mathcal{J}_{s ; \Omega}$ is not symmetric. Indeed, $J(4,0)=0$ and $J(0,4)=16$.

(I.2) $\left(U, Q_{s ; \Omega}\right)$ is a quasi- $b$-gauge space, and $\mathcal{J}_{s ; \Omega} \in \mathbb{J}_{\left(U, Q_{s ; \Omega}\right)}^{L} \cap \mathbb{J}_{\left(U, Q_{s ; \Omega}\right)}^{R}$. See Example 3.12.

(I.3) The property $T: U \rightarrow C l^{L-Q_{s ; \Omega}}(U)\left(T: U \rightarrow C l^{R-Q_{s ; \Omega}}(U)\right)$ holds. This follows from (3.32) and Definition 3.14 and 3.13(C).

(I.4) $T: U \rightarrow C l^{L-Q_{s ; \Omega}(U)}$ is a $\left(\mathcal{D}_{1}^{L-\mathcal{J}_{s ; \Omega}}, \mu=\frac{1}{10}\right)$-contraction on $U$, i.e., for all $u, v \in U$ $D_{1}^{L-\mathcal{J}_{s ; \Omega}}(T(u), T(v)) \leq \mu J(u, v)$, where $D_{1}^{L-\mathcal{J}_{s ; \Omega}}(U, V)=\max \left\{\sup _{u \in U} J(u, V), \sup _{v \in V} J(U, v)\right\}, U, V \in 2^{U}$.

Denoting $D_{1}^{L-\mathcal{J}_{s ; \Omega}}=D_{1}$, we prove this in the following subcases:

(I.4.1) If $u, v \in[0,3) \cup(3,6]$, this implies $u, v \in G, T(u)=T(v)=[4,6]=E \subset G$, and by (3.32) for all $e \in E$, we have $\inf \{J(e, f): f \in E\}=J(e, e)=q(e, e)=0$. Thus, $D_{1}(T(u), T(v))=0 \leq \mu J(u, v)$.

(I.4.2) If $u \in[0,3) \cup(3,6]$ and $v=3$, then $u \in G, v \notin G, J(u, v)=40, T(u)=[4,6]=E \subset G$, $T(v)=[5,6]=F \subset G$ and by (3.32), $e \in E$ suggests

$$
\inf \{J(e, f)=q(e, f): f \in F\}= \begin{cases}4 & \text { whenever } e \in[4 ; 5] \\ 0 & \text { whenever } e \in[5 ; 6] .\end{cases}
$$

Whereas, $f \in F$ inferred $\inf \{J(e, f)=q(e, f): e \in E\}=0$. Thus, $D_{1}(T(u), T(v))=4=\mu J(u, v)$.

(I.4.3) If $u=3$ and $v \in[0,3) \cup(3,6]$, then $u \notin G, v \in G, J(u, v)=40, T(u)=[5,6]=E \subset G$, $T(v)=[4,6]=F \subset G$. As a result, by (3.32), $e \in E$ implies $\inf \{J(e, f)=q(e, f): f \in F\}=0$. Further, by (3.32) $f \in F$ suggests $\inf \{J(e, f): e \in E\}=0$. Thus, $D_{1}(T(u), T(v))=0 \leq \mu J(u, v)$.

(I.4.4) If $u=v=3$, then $J(u, v)=36, T(u)=T(v)=[5,6]=E \subset G$ and for all $e \in E$ $\inf \{J(e, f)=q(e, f): f \in E\}=q(e, e)=0$. Therefore, $D_{1}(T(u), T(v))=0<\mu J(u, v)$.

(I.5) To prove that there exists $v \in T(u)$ such that $J(u, v)<J(u, T(u))+\gamma$, for all $u \in U$ and for all $\gamma \in(0, \infty)$, we observe the following subcases:

(I.5.1) If $u \in[0,3) \cup(3,4)$ and $v=4 \in T(u)=[4,6]$, then $J(u, v)=q(u, v)=(u-v)^{2}$, $J(u, T(u))=(u-v)^{2}$ and $J(u, v)<J(u, T(u))+\gamma$ for all $\gamma \in(0, \infty)$.

(I.5.2) If $u \in[4,6]$ and $v=4 \in T(u)=[4,6]$, then $J(u, v)=q(u, v)=0, J(u, T(u))=0$ and $J(u, v)<J(u, T(u))+\gamma$ for all $\gamma \in(0, \infty)$.

(I.5.3) If $u=3$ and $v \in T(u)=[5,6]$, then $J(u, v)=J(u, T(u))=40$ and $J(u, v)<J(u, T(u))+\gamma$ for all $\gamma \in(0, \infty)$. 
(I.6) Let $(U, T)$ be left $\mathcal{J}_{s ; \Omega}$-admissible at $U$. We show that if $z^{0} \in U$, and $\left\{z^{m}: m \in\{0\} \cup \mathbb{N}\right\}$ fulfils the properties

$$
z^{m+1} \in T\left(z^{m}\right), \quad \text { for all } m \in\{0\} \cup \mathbb{N}
$$

and

$$
\lim _{m \rightarrow \infty} \sup _{n>m} J\left(z^{m}, z^{n}\right)=0
$$

then

$$
\lim _{m \rightarrow \infty} J\left(z, z^{m}\right)=0 \quad \text { where } z=6
$$

In fact, we observe

$$
T^{[m]}(U)=[4 ; 6] \subset G \text { for } m \geq 2 .
$$

We can also write (3.36) in the form that there exists $m_{0} \in \mathbb{N}$ such that for all $\epsilon>0$ and for all $n>m \geq m_{0}$, we have $J\left(z^{m}, z^{n}\right)<\epsilon$ and so, in particular in view of (3.38), (3.32), and (3.33), this implies that there exists $m_{1} \geq m_{0}$ such that for all $0<\epsilon$ and for all $n>m \geq m_{1}$, we have

$$
J\left(z^{m}, z^{n}\right)=q\left(z^{m}, z^{n}\right)=0<\epsilon .
$$

From (3.38), (3.39), (3.32), and (3.33), we conclude that $z^{m} \geq z^{m+1}$ for all $m \geq m_{1}$, and since $6 \geq z^{m}$ for all $m$ and $6 \in G$, we have $\lim _{m \rightarrow \infty} q\left(z, z^{m}\right)=0$ where $z=6$ and this implies (3.37). Thus, $(U, T)$ is the left $\mathcal{J}_{s ; \Omega}$-admissible at $U$.

(I.7) To prove $(U, T)$ is a left $Q_{s ; \Omega}$-quasi-closed map in $U$, suppose $\left(w_{m}: m \in \mathbb{N}\right) \subset T(U)$ is a left $Q_{s ; \Omega}$-converging sequence in $U$. Now, as $[4,6] \subset C l^{L-Q_{s ; \Omega} \Omega}(U)$, there exists $w \in T(U)=[4,6]$ such that $\lim _{m \rightarrow \infty} q\left(w, w_{m}\right)=0$.

Equivalently, there exist $w \in T(U)=[4,6]$ and $m_{0}$ such that $q\left(w, w_{m}\right)<\epsilon$ for all $\epsilon>0$ and for all $m \geq m_{0}$, and thus, by (3.33) and (3.32), there exist $w \in T(U)=[4,6]$ and $m_{1} \geq m_{0}$ such that $q\left(w, w_{m}\right)=0<\epsilon$, for all $0<\epsilon$ and $m \geq m_{1}$, or analogously there exist $w \in T(U)=[4,6]$ and $m_{1}$ such that $w \geq w_{m}$ for all $m \geq m_{1}$. Obviously, then $[w, 6] \subset S_{\left(w_{m}: m \in \mathbb{N}\right)}^{L-Q_{s} \Omega}$. The consideration above implies that if $\left(x_{m}: m \in \mathbb{N}\right)$ and $\left(y_{m}: m \in \mathbb{N}\right)$ are fixed and arbitrary subsequences of $\left\{w_{m}: m \in \mathbb{N}\right\}$ fulfilling $y_{m} \in T\left(x_{m}\right)$ for all $m \in \mathbb{N}$, then there exists $m_{1}$ such that $x_{m} \in[4 ; 6] \wedge y_{m} \in T\left(x_{m}\right) \wedge z \geq x_{m} \wedge z \geq y_{m} \wedge z \in T(z)$ for all $m \geq m_{1}$ and for all $z \in[w, 6]$.

(I.8) From (I.1)-(I.7), we observe that all the hypotheses of Theorem 3.22 hold in the left case.

Thus, we have $\operatorname{Fix}(T)=[4 ; 6]$ and declare that if $z^{0} \in U, z^{1} \in T\left(z^{0}\right), z^{2} \in T\left(z^{1}\right)$ and $w \in[4 ; 6]$ are fixed and arbitrary and $z^{m}=w$ for all $m \geq 3$, then the sequence $\left\{z^{m}: m \in\{0\} \cup \mathbb{N}\right\}$, beginning at $z^{0}$ and left $Q_{s ; \Omega}$-converging to each point $z$, satisfies $z \in T(z)$. 
Remark 3.27 Let a quasi- $b$-gauge space $\left(U, Q_{s ; \Omega}\right)$ and a family $\mathcal{J}_{s ; \Omega}=\{J\}$ be as defined in Example 3.26.

(a) (3.32) implies that $q$ is a quasi-pseudo- $b$-metric, where $s=2$, and $q$ is not a quasi-pseudo-metric. This implies that $\left(U, Q_{s ; \Omega}\right)$ is a quasi- $b$-gauge space but not a quasi-gauge space. Hence a quasi- $b$-gauge space becomes a more general space than a quasi-gauge space.

(b) From cases I.4.2 and I.4.3, it follows that $4=D_{1}^{L-\mathcal{J}_{s ; \Omega}}(E, F) \neq D_{1}^{L-\mathcal{J}_{s ; \Omega}}(E, F)=0$ for $F=[5 ; 6]$ and $E=[4 ; 6]$.

(c) We see that $D_{1}^{L-\mathcal{J}_{s ; \Omega}}(E, E) \neq 0$ if $E=\{3\}$.

\section{Consequences and an application}

This section is concerned with some important consequences and an application of the obtained results. The following corollaries are some fascinating consequences of the main results.

Corollary 4.1 Let $(U, Q)$ be a quasi-gauge space, let $\mathcal{J}=\left\{J_{\beta}: \beta \in \Omega\right\}$ be a $L(R) \mathcal{J}$-family on $U$, and let $\zeta \in\{1,2,3\}$. Assume, moreover, that $\mu=\left\{\mu_{\beta}\right\}_{\beta \in \Omega} \in[0,1)$, and the map $T$ : $U \rightarrow C l^{L-\mathcal{J}}(U)\left(T: U \rightarrow C l^{R-\mathcal{J}}(U)\right)$ satisfies:

(i) $T$ is $\left(\mathcal{D}_{\zeta}^{L-\mathcal{J}}, \mu\right)$-contraction on $U\left(\left(\mathcal{D}_{\zeta}^{R-\mathcal{J}}, \mu\right)\right.$-contraction on $\left.U\right)$;

(ii) for any $u \in U$ and any $\gamma=\left\{\gamma_{\beta}\right\}_{\beta \in \Omega} \in(0, \infty)$, there exists $v \in T(u)$ such that for all $\beta \in \Omega$

$$
\begin{aligned}
& J_{\beta}(u, v)<J_{\beta}(u, T(u))+\gamma_{\beta}, \\
& \left(J_{\beta}(v, u)<J_{\beta}(T(u), u)+\gamma_{\beta}\right) .
\end{aligned}
$$

We have the following:

(I) If $(U, T)$ at a point $z^{0} \in U$ is $L(R) \mathcal{J}$-admissible, then there exists a sequence $\left\{z^{m}: m \in\{0\} \cup \mathbb{N}\right\}$ starting at $z^{0} \in U$ such that $z^{m} \in T\left(z^{m-1}\right)$ for all $m \in \mathbb{N}$, a point $z \in U$, and $r=\left\{r_{\beta}\right\}_{\beta \in \Omega} \in(0, \infty)$ such that $\left.z^{m} \in B^{L-\mathcal{J}}\left(z^{0}, r\right)\left(z^{m} \in B^{R-\mathcal{J}}\left(z^{0}, r\right)\right\}\right)$ for all $m \in \mathbb{N}$ and $\lim _{m \rightarrow \infty}^{L-\mathcal{J}} z_{m}=z\left(\lim _{m \rightarrow \infty}^{R-\mathcal{J}} z_{m}=z\right)$.

(II) If $(U, T)$ at a point $z^{0} \in U$ is $L(R) \mathcal{J}$-admissible, and if $T^{[k]}$ is $L(R) Q$-quasi-closed map on $U$ for some $k \in \mathbb{N}$, then $\operatorname{Fix}\left(T^{[k]}\right)$ is non-empty and there exists a sequence $\left\{z^{m}: m \in\{0\} \cup \mathbb{N}\right\}$ starting at $z^{0} \in U$ such that $z^{m} \in T\left(z^{m-1}\right)$ for all $m \in \mathbb{N}$, a point $z \in \operatorname{Fix}\left(T^{[k]}\right)$, and $r=\left\{r_{\beta}\right\}_{\beta \in \Omega} \in(0, \infty)$ such that $z^{m} \in B^{L-\mathcal{J}}\left(z^{0}, r\right)\left(z^{m} \in B^{R-\mathcal{J}}\left(z^{0}, r\right)\right)$ for all $m \in \mathbb{N}$ and $\lim _{m \rightarrow \infty}^{L-\mathcal{J}} z_{m}=z\left(\lim _{m \rightarrow \infty}^{R-\mathcal{J}} z_{m}=z\right)$.

Proof The proof follows easily by taking $s_{\beta}=1$ for each $\beta \in \Omega$ in the proof of Theorem 3.21.

Corollary 4.2 Let $(U, Q)$ is a quasi-gauge space, let $\mathcal{J}=\left\{J_{\beta}: \beta \in \Omega\right\}$ is a $L(R) \mathcal{J}$-family of generalized quasi-pseudo distances on $U$, and let $\zeta \in\{1,2\}$. Moreover, assume that $\mu=$ $\left\{\mu_{\beta}\right\}_{\beta \in \Omega} \in[0,1)$ and $T: U \rightarrow U$ be $\left(\mathcal{D}_{\zeta}^{L-\mathcal{J}}, \mu\right)$-contraction on $U\left(\left(\mathcal{D}_{\zeta}^{R-\mathcal{J}}, \mu\right)\right.$-contraction on $U)$. We have the following:

(I) If $(U, T)$ at a point $z^{0} \in U$ is $L(R) \mathcal{J}$-admissible, then there exists a sequence $\left\{z^{m}: m \in\{0\} \cup \mathbb{N}\right\}$ starting at $z^{0} \in U$ such that $\left(z^{m}=T^{[m]}\left(z^{0}\right): m \in\{0\} \cup \mathbb{N}\right)$, a point $z \in U$, and $r=\left\{r_{\beta}\right\}_{\beta \in \Omega} \in(0, \infty)$ such that $z^{m} \in B^{L-\mathcal{J}}\left(z^{0}, r\right)\left(z^{m} \in B^{R-\mathcal{J}}\left(z^{0}, r\right)\right)$ for all $m \in \mathbb{N}$ and $\lim _{m \rightarrow \infty}^{L-\mathcal{J}} z_{m}=z\left(\lim _{m \rightarrow \infty}^{R-\mathcal{J}} z_{m}=z\right)$. 
(II) If $(U, T)$ at a point $z^{0} \in U$ is $L(R) \mathcal{J}$-admissible, and if $T^{[k]}$ is $L(R) Q$-quasi-closed map on $U$ for some $k \in \mathbb{N}$, then $\operatorname{Fix}\left(T^{[k]}\right)$ is non-empty and there exists a sequence $\left\{z^{m}: m \in 0 \cup \mathbb{N}\right\}$ starting at $z^{0} \in U$ such that $\left(z^{m}=T^{[m]}\left(z^{0}\right): m \in\{0\} \cup \mathbb{N}\right)$, a point $z \in \operatorname{Fix}\left(T^{[k]}\right)$, and $r=\left\{r_{\beta}\right\}_{\beta \in \Omega} \in(0, \infty)$ such that $z^{m} \in B^{L-\mathcal{J}}\left(z^{0}, r\right)\left(z^{m} \in B^{R-\mathcal{J}}\left(z^{0}, r\right)\right)$ for all $m \in \mathbb{N}, \lim _{m \rightarrow \infty}^{L-\mathcal{J}} z_{m}=z\left(\lim _{m \rightarrow \infty}^{R-\mathcal{J}} z_{m}=z\right)$, and we have

$$
J_{\beta}(z, T(z))=J_{\beta}(T(z), z)=0
$$

for all $\beta \in \Omega$ and for all $z \in \operatorname{Fix}\left(T^{[k]}\right)$.

(III) If $(U, Q)$ is a Hausdorff space, if $(U, T)$ at a point $z^{0} \in U$ is $L(R) \mathcal{J}$-admissible, and if $T^{[k]}$ is $L(R) Q$-quasi-closed map on $U$ for some $k \in \mathbb{N}$, then there exists a sequence $\left\{z^{m}: m \in 0 \cup \mathbb{N}\right\}$ starting at $z^{0} \in U$ such that $\left(z^{m}=T^{[m]}\left(z^{0}\right): m \in\{0\} \cup \mathbb{N}\right)$, a point $z \in \operatorname{Fix}\left(T^{[k]}\right)=\operatorname{Fix}(T)=\{z\}$, and $r=\left\{r_{\beta}\right\}_{\beta \in \Omega} \in(0, \infty)$ such that $z^{m} \in B^{L-\mathcal{J}}\left(z^{0}, r\right)\left(z^{m} \in B^{R-\mathcal{J}}\left(z^{0}, r\right)\right)$ for all $m \in \mathbb{N}, \lim _{m \rightarrow \infty}^{L-\mathcal{J}} z_{m}=z\left(\lim _{m \rightarrow \infty}^{R-\mathcal{J}} z_{m}=z\right)$, and we have

$$
J_{\beta}(z, z)=0 \quad \text { for all } \beta \in \Omega
$$

Proof The proof easily follows by taking $s_{\beta}=1$ for each $\beta \in \Omega$ in the proof of Theorem 3.23.

\section{Remark 4.3}

(a) We note that Corollary 4.2 corresponds to Theorem 11.1 of Wlodarczyk and Plebaniak [41]. Hence, our Theorem 3.24 is a generalization of their result.

(b) The proof of the fixed point theorem due to Banach [22] and Nadler [23] requires the completeness of the spaces $(U, q)$ and $\left(C B(U), H^{q}\right)$, the continuity of $q$ and $H^{q}$ and the continuity of the mapping $T$. Our main theorems corresponding to Theorem 3.22 and Theorem 3.24 do not use these assumptions and leave the assertion more general. Hence, our results are a new generalization of the fixed point theorem due to Banach and Nadler.

Now, we present an application on the existence of a solution of an integral equation. For this, consider the Volterra integral equation in the form

$$
x(t)=f(t)+\eta \int_{0}^{t} K(t, s, x(s)) d s, \quad t \in[0, \infty)
$$

which is an integral equation located in the space $C[0, \infty)$, i.e., the space of all continuous functions defined on the interval $I=[0, \infty)$, where $f: I \rightarrow \mathbb{R}$ is a continuous function, $K: I \times I \times \mathbb{R} \rightarrow \mathbb{R}$ is a continuous and nondecreasing function, and $\eta \in[0,1)$.

Let $U=(C[0, \infty), \mathbb{R})$. Define the quasi-pseudo- $b$-metric for all $x, y \in U$ by

$$
q_{m}(x, y)= \begin{cases}\|(x-y)\|_{m} & \text { if } x \neq y \\ 0 & \text { if } x=y\end{cases}
$$

where $\|x\|_{m}=\max _{r \in[0, m]}(x(r))^{2}$, for all $x \in U$, with $m \in \mathbb{N}$.

Clearly, $Q_{s ; \mathbb{N}}=\left\{q_{m}: m \in \mathbb{N}\right\}$ is a quasi- $b$-gauge on $U$, and thus $\left(U, Q_{s ; \mathbb{N}}\right)$ is a quasi- $b$-gauge space, which is complete and the Hausdorff one. Here, in particular we take $Q_{s ; \mathbb{N}}=\mathcal{J}_{s ; \mathbb{N}}$. 
Theorem 4.4 Suppose that the following statements hold:

(i) for each $t, s \in[0, m]$ and $x, y \in U$, there exists a continuous mapping $g: I \times I \rightarrow I$ such that $|K(t, s, x(s))-K(t, s, y(s))| \leq \sqrt{g(t, s) q_{m}(x, y)}$ for each $m \in \mathbb{N}$;

(ii) $\sup _{t \geq 0} \int_{0}^{t} \sqrt{g(t, s)} d s=b<1$;

(iii) $T$ is $Q_{s ; \mathbb{N}}$-quasi-closed map on $U$.

Then the integral equation (4.3) has at least one solution.

Proof Define $T: C[0, \infty) \rightarrow C[0, \infty)$ as follows

$$
T x(t)=f(t)+\eta \int_{0}^{t} K(t, s, x(s)) d s, \quad t \in[0, \infty) .
$$

For any $x, y \in U$ and $t \in[0, m]$, consider

$$
\begin{aligned}
(T x(t)-T y(t))^{2} & =\left(f(t)+\eta \int_{0}^{t} K(t, s, x(s)) d s-\left(f(t)+\eta \int_{0}^{t} K(t, s, y(s)) d s\right)\right)^{2} \\
& =\left(\eta \int_{0}^{t} K(t, s, x(s)) d s-\eta \int_{0}^{t} K(t, s, y(s)) d s\right)^{2} \\
& =\eta^{2}\left(\int_{0}^{t}|K(t, s, x(s))-K(t, s, y(s))| d s\right)^{2} \\
& \leq \eta^{2}\left(\int_{0}^{t} \sqrt{g(t, s) q_{m}(x, y)} d s\right)^{2} \\
& \leq \eta^{2}\left(\int_{0}^{t} \sqrt{g(t, s)} d s\right)^{2} q_{m}(x, y) \\
& =\eta^{2} b^{2} q_{m}(x, y) \\
& =\mu q_{m}(x, y), \quad \text { where } \mu=\eta^{2} b^{2}<1 .
\end{aligned}
$$

Hence, for each $x, y \in U$ such that $T x \neq T y$ and $m \in \mathbb{N}$, we obtain

$$
q_{m}(T x, T y) \leq \mu q_{m}(x, y) \quad \text { where } \mu<1 .
$$

For $T x=T y$, we have $q_{m}(T x, T y)=0$, so (4.6) holds. Hence, by Theorem (3.24), the operator $T$ has a fixed point, that is, the integral equation (4.3) has at least one solution.

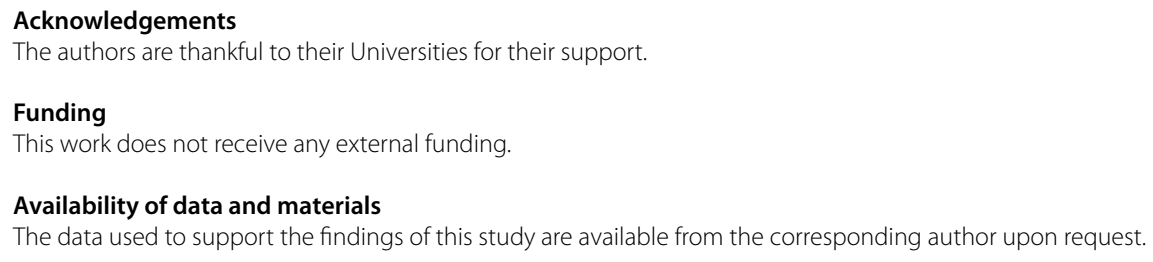

\section{Declarations}




\section{Author details}

${ }^{1}$ Department of Mathematics, Quaid-i-Azam University, Islamabad, Pakistan. ${ }^{2}$ Institut Supérieur d'Informatique et des Techniques de Communication, Université de Sousse, H. Sousse, 4000, Tunisia. ${ }^{3}$ China Medical University Hospital, China Medical University, Taichung 40402, Taiwan. ${ }^{4}$ Department of Mathematics and Applied Mathematics, Sefako Makgatho Health Sciences University, Ga-Rankuwa, South Africa. ${ }^{5}$ Research Institute for Natural Sciences, Hanyang University, Seoul 04763, Korea.

\section{Publisher's Note}

Springer Nature remains neutral with regard to jurisdictional claims in published maps and institutional affiliations.

Received: 24 August 2021 Accepted: 28 December 2021 Published online: 07 January 2022

\section{References}

1. Dugundji, J.: Topology. Allyn and Bacon, Boston (1966)

2. Reily, I.L.: Quasi-gauge spaces. J. Lond. Math. Soc. 6, 481-487 (1973)

3. Ali, M.U., Kamran, T., Postolache, M.: Fixed point theorems for multivalued G-contractions in Housdorff $b$-gauge space. J. Nonlinear Sci. Appl. 8, 847-855 (2015)

4. Agarwal, R.P., Cho, Y.J., Regan, D.O.: Homotopy invariant results on complete gauge spaces. Bull. Aust. Math. Soc. 67, 241-248 (2003)

5. Frigon, M.: Fixed point results for generalized contractions in gauge spaces and applications. Proc. Am. Math. Soc. $128,2957-2965(2000)$

6. Chis, A., Precup, R.: Continuation theory for general contractions in gauge spaces. Fixed Point Theory Appl. 3, 173-185 (2004)

7. Chifu, C., Petrusel, G.: Fixed point results for generalized contractions on ordered gauge spaces with applications. Fixed Point Theory Appl. 2011, 10 (2011)

8. Lazara, T., Petrusel, G.: Fixed points for non-self operators in gauge spaces. J. Nonlinear Sci. Appl. 6, 29-34 (2013)

9. Cherichi, M., Samet, B., Vetro, C.: Fixed point theorems in complete gauge spaces and applications to second ordernonlinear initial value problems. J. Funct. Spaces Appl. 2013, Article ID 293101 (2013)

10. Cherichi, M., Samet, B.: Fixed point theorems on ordered gauge spaces with applications to nonlinear integral equations. Fixed Point Theory Appl. 2012, 19 (2012)

11. Jleli, M., Karapinar, E., Samet, B.: Fixed point results for a $\alpha-\psi_{\lambda}$-contractions on gauge spaces and applications. Abstr. Appl. Anal. 2013, 7 (2013)

12. Kada, O., Suzuki, T., Takahashi, W.: Nonconvex minimization theorems and fixed point theorems in complete metric spaces. Math. Jpn. 44, 381-391 (1996)

13. Suzuki, T.: Generalized distance and existence theorems in complete metric spaces. J. Math. Anal. Appl. 253, 440-458 (2001)

14. Lin, L.J., Du, W.S.: Ekeland's variational principle, minimax theorems and existence of nonconvex equilibria in complete metric spaces. J. Math. Anal. Appl. 323, 360-370 (2006)

15. Wlodarczyk, K., Plebaniak, R.: New completeness and periodic points of discontinuous contractions of Banach-type in quasi-gauge spaces without Hausdorff property. Fixed Point Theory Appl. 2013, Article ID 289 (2013)

16. Plebaniak, R.: New generalized pseudodistance and coincidence point theorem in a $b$-metric space. Fixed Point Theory Appl. 270, 1-20 (2013)

17. Plebaniak, R.: On best proximity points for set-valued contractions of Nadler type with respect to $b$-generalized pseudodistances in b-metric spaces. Fixed Point Theory Appl. 39, 1-13 (2014)

18. Wlodarczyk, K.: Set-valued leader type contractions, periodic point and end point theorems, quasi-triangular spaces, Bellman and Volterra equations. Fixed Point Theory Appl. 6, 1-54 (2020)

19. Wlodarczyk, K.: Periodic and fixed points of the leader type contractions in quasi-triangular sapces. Fixed Point Theory Appl. 85, 1-28 (2016)

20. Wlodarczyk, K.: Quasi-triangular, Pompeiu-Hausdorff quasi-distances, and periodic and fixed point theorems of Bannach and Nadler types. Abstr. Appl. Anal. 2015, 16 (2015)

21. Wlodarczyk, K., Plebaniak, R.: Quasigauge spaces with generalized quasipseudodistances and periodic points of dissipative set-valued dynamic system. Fixed Point Theory Appl. 2011, Article ID 712706 (2011)

22. Banach, S.: Sur les operations dans les ensembles abstraits et leurs applications aux équations intégrales. Fundam. Math. 3, 133-181 (1922)

23. Nadler, S.B.: Multi-valued contraction mappings. Pac. J. Math. 30, 475-488 (1969)

24. Aydi, H., Felhi, A., Karapina, E., Sahmim, S.: A Nadler-type fixed point theorem in dislocated spaces and applications. Miskolc Math. Notes 19, 111-124 (2018)

25. Karapinar, E., Khojasteh, F., Mitrovic, Z.D.: A proposal for revisting Banach and Caristi type theorems in $b$-metric spaces Mathematics 7(4), 308 (2019)

26. Kutbi, M.A., Karapinar, E., Ahmad, J., Azam, A.: Some fixed point results for multi-valued mappings in $b$-metric spaces J. Inequal. Appl. 2014, 126 (2014)

27. Bakhtin, I.A.: The contraction mapping principle in almost metric spaces. Funct. Anal. 30, 26-37 (1989)

28. Czerwik, S.: Contraction mappings in b-metric spaces. Acta Math. Inform. Univ. Ostrav. 1, 5-11 (1993)

29. Hussain, S.: Fixed point and common fixed point theorems on ordered cone b-metric space over Banach algebra. J. Nonlinear Sci. Appl. 13, 22-33 (2020)

30. Antal, S., Gairola, U.C.: Generalized Suzuki type $\alpha$-Z-contraction in b-metric space. J. Nonlinear Sci. Appl. 13, 212-222 (2020)

31. Mishra, L.N., Dewangan, V., Mishra, V.N., Karateke, S.: Best proximity points of admissible almost generalized weakly contractive mappings with rational expressions on b-metric spaces. J. Math. Comput. Sci. 22, 97-109 (2021)

32. Phiangsungnoen, S., Sintunavarat, W., Kumam, P.: Fixed point results, generalized Ulam-Hyers stability and well-posedness via alpha-admissible mappings in b-metric spaces. Fixed Point Theory Appl. 1, 1-17 (2014) 
33. Kumam, P., Sintunavarat, W.: The existence of fixed point theorems for partial q-set-valued quasi-contractions in b-metric spaces. Fixed Point Theory Appl. 1, 226 (2014)

34. Aydi, H., Bota, M.F., Karapinar, E., Moradi, S.: A common fixed point for weak $\phi$-contractions on $b$-metricspaces. Fixed Point Theory 13, 337-346 (2012)

35. Samreen, M., Kamran, T., Shahzad, N.: Some fixed point theorems in b-metric space endowed with graph. Abstr. Appl. Anal. 2013, 967132 (2013)

36. Ameer, E., Aydi, H., Arshad, M., Alsamir, H., Noorani, M.S.: Hybrid multivalued type contraction mappings in $\alpha_{K}$-complete partial b-metric spaces and applications. Symmetry 11(1), 86 (2019)

37. Karapinar, E., Czerwik, S., Aydi, H.: $(\alpha, \psi)$-Meir-Keeler contraction mappings in generalized b-metric spaces. J. Funct. Spaces 2018, Article ID 3264620 (2018)

38. Kir, N., Kiziltun, H.: On some well known fixed point theorems in b-metric spaces. Turk. J. Anal. Number Theory 1, 13-16 (2013)

39. Kamran, T., Samreen, M., Ain, Q.U.: A generalization of $b$-metric space and some fixed point theorems. Mathematics 5 , 1-7 (2017)

40. Kelly, J.C.: Bitopological spaces. Proc. Lond. Math. Soc. 13, 71-89 (1963)

41. Wlodarczyk, K.: Hausdorff quasi-distances, periodic and fixed points for Nadler type set-valued contractions in quasi-gauge spaces. Fixed Point Theory Appl. 2014, Article ID 239 (2014)

\section{Submit your manuscript to a SpringerOpen ${ }^{\circ}$ journal and benefit from:}

- Convenient online submission

Rigorous peer review

- Open access: articles freely available online

- High visibility within the field

- Retaining the copyright to your article

Submit your next manuscript at $\boldsymbol{\nabla}$ springeropen.com 